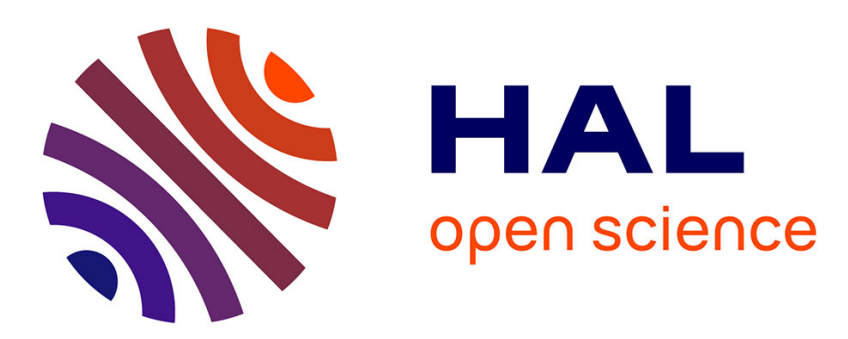

\title{
Random matrix theory and non-parametric model of random uncertainties in vibration analysis
}

Christian Soize

\section{To cite this version:}

Christian Soize. Random matrix theory and non-parametric model of random uncertainties in vibration analysis. Journal of Sound and Vibration, 2003, 263 (4), pp.893-916. 10.1016/S0022460X(02)01170-7 . hal-00686213

\section{HAL Id: hal-00686213 \\ https://hal.science/hal-00686213}

Submitted on 8 Apr 2012

HAL is a multi-disciplinary open access archive for the deposit and dissemination of scientific research documents, whether they are published or not. The documents may come from teaching and research institutions in France or abroad, or from public or private research centers.
L'archive ouverte pluridisciplinaire HAL, est destinée au dépôt et à la diffusion de documents scientifiques de niveau recherche, publiés ou non, émanant des établissements d'enseignement et de recherche français ou étrangers, des laboratoires publics ou privés. 
TITLE: Random matrix theory and non-parametric model of random uncertainties in vibration analysis

AUTHOR: C. Soize

PROFESSIONAL ADDRESS:

Laboratoire de Mécanique - LaM

Université de Marne-la-Vallée

5 Bd Descartes

77454 Marne-la-Vallée Cedex 2

France

E-MAIL: soize@univ-mlv.f

TOTAL NUMBER OF PAGES: 32 
Recently, a new approach, called a non-parametric model of random uncertainties, has been introduced for modelling random uncertainties in linear and non-linear elastodynamics in the low-frequency range. This non-parametric approach differs from the parametric methods for random uncertainties modelling and has been developed in introducing a new ensemble of random matrices constituted of symmetric positive-definite real random matrices. This ensemble differs from the GOE and from the other known ensembles of the random matrix theory. The present paper has three main objectives. The first one is to study the statistics of the random eigenvalues of random matrices belonging to this new ensemble and to compare with the GOE. The second one is to compare this new ensemble of random matrices with the GOE in the context of the non-parametric approach of random uncertainties in structural dynamics for the low-frequency range. The last objective is to give a new validation for the non-parametric model of random uncertainties in structural dynamics in comparing, in the low-frequency range, the dynamical response of a simple system having random uncertainties modelled by the parametric and the non-parametric methods. These three objectives will allow us to conclude about the validity of the different theories. 


\section{INTRODUCTION}

The random matrix theory were introduced and developed in mathematical statistics by Wishart and others in the 1930s and was intensively studied by physicists and mathematicians in the context of nuclear physics. These works began with Wigner [1] in the 1950s and received an important effort in the 1960s by Wigner, Dyson, Mehta [2-4] and others. In 1965, Poter [5] published a volume of important papers in this field, followed, in 1967 by the first edition of the Mehta book [6] whose second edition [7] published in 1991 is an excellent synthesis of the random matrix theory. For physical applications, the most important ensemble of the random matrix theory, is the Gaussian Orthogonal Ensemble (GOE) for which the elements are constituted of real symmetric random matrices with statistically independent entries and which are invariant under orthogonal linear transformations.

The random matrix theory has been used in other domains that nuclear physics. In 1984 and 1986, Bohigas at al $[8,9]$ found that the level fluctuations of the quantum Sinai's billard were able to be predicted with the GOE of random matrices. In 1989, Weaver [10] show that the higher frequencies of elastodynamic structures constituted of small aluminium blocks have the behavior of the eigenvalues of a matrix belonging to the GOE. Then, Legrand, Schmit and Sornette [11-14] studied the high-frequency spectral statistics with the GOE for elastodynamics and vibration problems in the high-frequency range. More recently, Langley [15] show that the system of natural frequencies in the high-frequency range of linear uncertain dynamic systems is a non-Poisson point-process. All these results have clearly been validated for the high-frequency range in elastodynamics but not at all for the low- and medium-frequency ranges.

Recently, a new approach, called a non-parametric model of random uncertainties, has been introduced [16-20] for modelling random uncertainties in linear and non-linear elastodynamics in the modal range, that is to say, in the low-frequency range. This non-parametric approach differs from the parametric [21-24] and stochastic finite element [25-30] methods for random uncertainties modelling and has been developed in introducing a new ensemble of random matrices constituted of symmetric positive-definite real random matrices $[16,18]$. This ensemble differs from the GOE and from the other known ensembles of the random matrix theory. This new ensemble is constructed using the maximum entropy principle [31-34] which allows the probability distribution of positive symmetric real random matrices to be constructed using only the available information. In order to improve the readability of this paper, one recalls fundamentals of the non-parametric model of random uncertainties introduced in the papers mentioned above. 
The present paper has three main objectives. The first one is to study the statistics of the random eigenvalues of random matrices belonging to this new ensemble of symmetric positive-definite real random matrices. This part will allow some properties of this new ensemble to be given and to be compared to those of the GOE. The second one is to compare these two ensembles of random matrices in the context of the non-parametric approach of random uncertainties in dynamic systems for the low-frequency range. This comparison will be limited to the case for which only the generalized stiffness matrix of the dynamic system is random, the generalized mass and damping matrices being deterministic. This limitation is due to the fact that, in the state of the art, the GOE does not allow a damped dynamic system to be modelled while the new ensemble allows mass, damping and stiffness random uncertainties to be modelled. Finally, the last objective of this paper is to give a new validation for the non-parametric model of random uncertainties in fixed dynamic systems in comparing, in the low-frequency range, the dynamical response of a simple system having random uncertainties modelled by the parametric and the non-parametric methods. It should be noted that the extension to the free dynamic systems having rigid body modes is straightforward using the additional developments introduced in Ref. [16] for the semi-positive definite real random matrices (instead of the positive-definite set of random matrices). These three objectives will allow us to conclude about the validity of the different theories and in particular, about the non-parametric modelling of random uncertainties in dynamic systems.

\subsection{BRIEF REVIEW ON THE NON-PARAMETRIC MODEL OF RANDOM UNCERTAINTIES IN VIBRATION} ANALYSIS

In this paper, $\mathbb{M}_{n}(\mathbb{R}), \mathbb{M}_{n}^{S}(\mathbb{R})$ and $\mathbb{M}_{n}^{+}(\mathbb{R})$ are the set of all the $(n \times n)$ real matrices, the set of all the symmetric $(n \times n)$ real matrices and the set of all the positive-definite symmetric $(n \times n)$ real matrices, respectively. One has $\mathbb{M}_{n}^{+}(\mathbb{R}) \subset \mathbb{M}_{n}^{S}(\mathbb{R}) \subset \mathbb{M}_{n}(\mathbb{R})$. If $[A]$ belongs to $M_{n}(\mathbb{R})$, $\|[A]\|_{F}=\left(\operatorname{tr}\left\{[A][A]^{T}\right\}\right)^{1 / 2}$ is the Frobenius norm of matrix $[A]$, where tr is the trace of the matrices, det is the determinant of the matrices and $[A]^{T}$ is the transpose of matrix $[A]$. The indicatrix function $\mathbb{1}_{\mathcal{B}}(b)$ of any set $\mathcal{B}$ is such that $\mathbb{1}_{\mathcal{B}}(b)$ is equal to 1 if $b \in \mathcal{B}$ and is equal to zero if $b \notin \mathcal{B}$. The gamma function is defined for $z>0$ by $\Gamma(z)=\int_{0}^{+\infty} t^{z-1} e^{-t} d t$. All the random variables are defined on a probability space $(\mathcal{A}, \mathcal{T}, \mathcal{P})$ and $E$ is the mathematical expectation.

In this introduction, one briefly recalls the main ideas introduced in references [16-18] concerning the non-parametric model in elastodynamics and vibrations for the low-frequency range and one limits the developments to the case of linear dynamic systems. 
The two main assumptions introduced to construct such a non-parametric model of random uncertainties in linear structural dynamics are:

(1) not using the local parameters of the boundary value problem modelling the dynamic system, but using the generalized coordinates directly related to dynamics (non-parametric approach);

(2) using the available information which is constituted of the mean reduced model constructed with the $n$ generalized coordinates of the mode-superposition method associated with the elastic modes corresponding to the $n$ lowest eigenfrequencies of the linear dynamic system assumed to be fixed, damped and stable.

To satisfy these two main assumptions, the non-parametric probabilistic model of random uncertainties consists in replacing the generalized diagonal mass matrix $\left[\underline{M}_{n}\right] \in \mathbb{M}_{n}^{+}(\mathbb{R})$, the generalized full damping matrix $\left[\underline{D}_{n}\right] \in \mathbb{M}_{n}^{+}(\mathbb{R})$ and the generalized diagonal stiffness matrix $\left[\underline{K}_{n}\right] \in \mathbb{M}_{n}^{+}(\mathbb{R})$ of the mean reduced model by the full random matrices $\left[\mathbf{M}_{n}\right],\left[\mathbf{D}_{n}\right]$ and $\left[\mathbf{K}_{n}\right]$ respectively, with values in $\mathbb{M}_{n}^{+}(\mathbb{R})$. The probability model of each random matrix $\left[\mathbf{M}_{n}\right],\left[\mathbf{D}_{n}\right]$ and $\left[\mathbf{K}_{n}\right]$ is constructed using the entropy optimization principle [32-34] from information theory [31], using only the available information. For instance, let us consider random matrix $\left[\mathbf{K}_{n}\right]$ for which the available information is constituted of the following constraints.

(C1) The mean value $E\left\{\left[\mathbf{K}_{n}\right]\right\}$ of $M_{n}^{+}(\mathbb{R})$-valued random matrix $\left[\mathbf{K}_{n}\right]$ is known and is equal to the corresponding generalized matrix $\left[\underline{K}_{n}\right] \in \mathbb{M}_{n}^{+}(\mathbb{R})$ of the mean reduced model,

$$
E\left\{\left[\mathbf{K}_{n}\right]\right\}=\left[\underline{K}_{n}\right] \in \mathbb{M}_{n}^{+}(\mathbb{R})
$$

(C2) The second-order moment $E\left\{\left\|\left[\mathbf{K}_{n}\right]^{-1}\right\|_{F}^{2}\right\}$ of the Frobenius norm of the inverse of random matrix $\left[\mathbf{K}_{n}\right]$ has to be finite

$$
E\left\{\left\|\left[\mathbf{K}_{n}\right]^{-1}\right\|_{F}^{2}\right\}<+\infty
$$

Random matrix $\left[\mathbf{K}_{n}\right]$ has to be with values in $\mathbb{M}_{n}^{+}(\mathbb{R})$ in order to obtain a mechanical system with random uncertainties, which models a fixed and stable dynamic system. For instance if there were uncertainties in the generalized mass matrix, the probability distribution should be such that this random generalized mass matrix be positive definite. If not, the probability model would be wrong because the generalized mass matrix of any dynamic system has to be positive definite. It seems natural to introduce constraint (C1). Constraint (C2) is absolutely necessary and allows a unique second-order random response of the dynamic system with random uncertainties to exist as proved in references [18,20]. 
It should be noted that such a non-parametric model of random uncertainties,

(1) allows the uncertainties for the parameters of the elastodynamic model to be taken into account (similarly to the parametric approaches, but using a global approach),

(2) but also, allows the model uncertainties to be taken into account, that is to say, modelling the errors which cannot be reached through the model parameters (by definition, any parametric approach cannot model the kind of uncertainties which correspond to non existing parameters in the boundary value problem under consideration); for instance, the use of the thick plate theory instead of the three-dimensional elasticity, etc.

\subsection{SUMMARIZING THE PROBABILITY MODEL FOR SYMMETRIC POSITIVE-DEFINITE REAL RAN-}

\section{DOM MATRICES}

In this subsection, one summarizes a part of the results developed in references [16-18], concerning the construction of the probability model for random matrix $\left[\mathbf{K}_{n}\right]$ with values in $M_{n}^{+}(\mathbb{R})$ using the entropy optimization principle for which the available information is defined by Eqs. (1) and (2). This ensemble of random matrices has been developed for the non-parametric approach of random uncertainties in the vibration analysis of dynamic systems.

\section{A. Normalization and dispersion parameter of random matrix $\left[\mathbf{K}_{n}\right]$}

Since $\left[\underline{K}_{n}\right]$ is a positive-definite real matrix, there is an upper triangular matrix $\left[\underline{L}_{K_{n}}\right]$ in $\mathbb{M}_{n}(\mathbb{R})$ (Cholesky factorization) such that

$$
\left[\underline{K}_{n}\right]=\left[\underline{L}_{K_{n}}\right]^{T}\left[\underline{L}_{K_{n}}\right]
$$

Considering Eq. (3), random matrix $\left[\mathbf{K}_{n}\right]$ can be written as

$$
\left[\mathbf{K}_{n}\right]=\left[\underline{L}_{K_{n}}\right]^{T}\left[\mathbf{G}_{K_{n}}\right]\left[\underline{L}_{K_{n}}\right],
$$

in which matrix $\left[\mathbf{G}_{K_{n}}\right]$ is a random variable with values in $\mathbb{M}_{n}^{+}(\mathbb{R})$ such that

$$
\left[\underline{G}_{K_{n}}\right]=E\left\{\left[\mathbf{G}_{K_{n}}\right]\right\}=\left[I_{n}\right],
$$

in which $\left[I_{n}\right]$ is the $(n \times n)$ identity matrix. Let $\delta_{K}>0$ be the real parameter defined by

$$
\delta_{K}=\left\{\frac{E\left\{\left\|\left[\mathbf{G}_{K_{n}}\right]-\left[\underline{G}_{K_{n}}\right]\right\|_{F}^{2}\right\}}{\left\|\left[\underline{G}_{K_{n}}\right]\right\|_{F}^{2}}\right\}^{1 / 2} .
$$

Parameter $\delta_{K}$ allows the dispersion of the probability model of random matrix $\left[\mathbf{K}_{n}\right]$ to be controlled. If $n_{0} \geq 1$ is a given and fixed integer, then the dispersion of the probability 
model is defined by giving parameter $\delta_{K}$, independent of $n$, a value such that $0<\delta_{K}<$ $\left\{\left(n_{0}+1\right) /\left(n_{0}+5\right)\right\}^{1 / 2}$. This upper bound for $\delta_{K}$ comes from the theory, is necessary for that Eq. (2) holds and is not a severe limitation for applications. In general, dimension $n$ of the reduced matrix model is high, greater than 10 or 100 . For instance, if $n$ is greater than 10 , $n_{0}$ can be chosen as $n_{0}=10$ and consequently, this upper bound is 0.856 which corresponds to a very high level of uncertainties which is generally not reached in the applications.

\section{B. Probability distribution and second-order moments of random matrix $\left[\mathbf{G}_{K_{n}}\right]$}

The probability distribution $P_{\left[\mathbf{G}_{K_{n}}\right]}$ of random matrix $\left[\mathbf{G}_{K_{n}}\right]$ is defined by a probability density function $\left[G_{n}\right] \mapsto p_{\left[\mathbf{G}_{K_{n}}\right]}\left(\left[G_{n}\right]\right)$ from $\mathbb{M}_{n}^{+}(\mathbb{R})$ into $\mathbb{R}^{+}=[0,+\infty[$, with respect to the measure (volume element) $\widetilde{d} G_{n}$ on the set $\mathrm{M}_{n}^{S}(\mathbb{R})$ of all the $(n \times n)$ real symmetric matrices defined by

$$
\widetilde{d} G_{n}=2^{n(n-1) / 4} \Pi_{1 \leq i \leq j \leq n} d\left[G_{n}\right]_{i j}
$$

One then has $P_{\left[\mathbf{G}_{K_{n}}\right]}=p_{\left[\mathbf{G}_{K_{n}}\right]}\left(\left[G_{n}\right]\right) \widetilde{d} G_{n}$ with the normalization condition

$$
\int_{\mathbb{M}_{n}^{+}(\mathbb{R})} p_{\left[\mathbf{G}_{K_{n}}\right]}\left(\left[G_{n}\right]\right) \widetilde{d} G_{n}=1 .
$$

Probability density function $p_{\left[\mathbf{G}_{K_{n}}\right]}\left(\left[G_{n}\right]\right)$ is then written as

$$
\begin{aligned}
p_{\left[\mathbf{G}_{K_{n}}\right]}\left(\left[G_{n}\right]\right)= & \mathbb{1}_{\mathbb{M}_{n}^{+}(\mathbb{R})}\left(\left[G_{n}\right]\right) \times C_{\mathbf{G}_{K_{n}}} \times\left(\operatorname{det}\left[G_{n}\right]\right)^{\left(1-\delta_{K}^{2}\right)\left(2 \delta_{K}^{2}\right)^{-1}(n+1)} \\
& \times \exp \left\{-(n+1)\left(2 \delta_{K}^{2}\right)^{-1} \operatorname{tr}\left[G_{n}\right]\right\}
\end{aligned}
$$

in which positive constant $C_{\mathbf{G}_{K_{n}}}$ is such that

$$
C_{\mathbf{G}_{K_{n}}}=\frac{(2 \pi)^{-n(n-1) / 4}\left(\frac{n+1}{2 \delta_{K}^{2}}\right)^{n(n+1)\left(2 \delta_{K}^{2}\right)^{-1}}}{\left\{\Pi_{j=1}^{n} \Gamma\left(\frac{n+1}{2 \delta_{K}^{2}}+\frac{1-j}{2}\right)\right\}} .
$$

The covariance $C_{j k, j^{\prime} k^{\prime}}^{G_{K_{n}}}$ of random variables $\left[\mathbf{G}_{K_{n}}\right]_{j k}$ and $\left[\mathbf{G}_{K_{n}}\right]_{j^{\prime} k^{\prime}}$, defined by

$$
C_{j k, j^{\prime} k^{\prime}}^{G_{K_{n}}}=E\left\{\left(\left[\mathbf{G}_{K_{n}}\right]_{j k}-\left[\underline{G}_{K_{n}}\right]_{j k}\right)\left(\left[\mathbf{G}_{K_{n}}\right]_{j^{\prime} k^{\prime}}-\left[\underline{G}_{K_{n}}\right]_{j^{\prime} k^{\prime}}\right)\right\}
$$

is written as

$$
C_{j k, j^{\prime} k^{\prime}}^{G_{K_{n}}}=\frac{\delta_{K}^{2}}{n+1}\left\{\delta_{j^{\prime} k} \delta_{j k^{\prime}}+\delta_{j j^{\prime}} \delta_{k k^{\prime}}\right\}
$$

where $\delta_{j k}=0$ if $j \neq k$ and $\delta_{j j}=1$. In particular, the variance of random variable $\left[\mathbf{G}_{K_{n}}\right]_{j k}$ is such that

$$
V_{j k}^{G_{K_{n}}}=\frac{\delta_{K}^{2}}{n+1}\left(1+\delta_{j k}\right)
$$


Let $\mathbf{b}=\left(b_{1}, \ldots, b_{n}\right)$ be any vector in $\mathbb{R}^{n}$. Its Euclidean norm $\|\mathbf{b}\|$ is such that $\|\mathbf{b}\|^{2}=$ $b_{1}^{2}+\ldots+b_{n}^{2}$. For $\theta$ fixed in $\mathcal{A}$, the realization $\left[\mathbf{G}_{K_{n}}(\theta)\right]^{-1}$ of random matrix $\left[\mathbf{G}_{K_{n}}\right]^{-1}$ is a matrix belonging to $\mathbf{M}_{n}^{+}(\mathbb{R})$ and $\left[\mathbf{G}_{K_{n}}(\theta)\right]^{-1} \mathbf{b}$ is a vector in $\mathbb{R}^{n}$ whose Euclidean norm is $\left\|\left[\mathbf{G}_{K_{n}}(\theta)\right]^{-1} \mathbf{b}\right\|$. One introduces the usual matrix norm $\left\|\left|\left[\mathbf{G}_{K_{n}}(\theta)\right]^{-1} \|\right|\right.$ of matrix $\left[\mathbf{G}_{K_{n}}(\theta)\right]^{-1}$ defined by

$$
\left\|\left|\left[\mathbf{G}_{K_{n}}(\theta)\right]^{-1}\right|\right\|=\max _{\mathbf{b} \in \mathbb{R}^{n},\|\mathbf{b}\|=1}\left\|\left[\mathbf{G}_{K_{n}}(\theta)\right]^{-1} \mathbf{b}\right\| .
$$

Consequently, |||[ $\left.\mathbf{G}_{K_{n}}(\theta)\right]^{-1}|| \mid$ is the Euclidean norm of the largest vector obtained by applying $\left[\mathbf{G}_{K_{n}}(\theta)\right]^{-1}$ to any vector with a unit Euclidean norm. One then has the following inequality,

$$
\forall n \geq n_{0} \quad, \quad E\left\{\left|\left\|\left[\mathbf{G}_{K_{n}}\right]^{-1} \mid\right\|^{2}\right\} \leq C_{\delta_{K}}<+\infty \quad\right.
$$

in which $C_{\delta_{K}}$ is a positive finite constant that is independent of $n$ but that depends on $\delta_{K}$. The property defined by Eq. (15) is fundamental to prove the convergence of the random response of the dynamic system with random uncertainties as dimension $n$ goes to infinity as proved in reference [18].

Let $\left[\mathbf{G}_{K_{n}}^{\prime}\right]$ be the random matrix with values in $M_{n}^{+}(\mathbb{R})$ defined by $\left[\mathbf{G}_{K_{n}}^{\prime}\right]=\left[\Psi_{n}\right]^{T}\left[\mathbf{G}_{K_{n}}\right]\left[\Psi_{n}\right]$ in which $\left[\Psi_{n}\right]$ is any real orthogonal matrix belonging to $\mathbb{M}_{n}(\mathbb{R})$. One then has

$$
p_{\left[\mathbf{G}_{K_{n}}^{\prime}\right]}\left(\left[G_{n}^{\prime}\right]\right) \widetilde{d} G_{n}^{\prime}=p_{\left[\mathbf{G}_{K_{n}}\right]}\left(\left[G_{n}^{\prime}\right]\right) \widetilde{d} G_{n}^{\prime}
$$

which proves the invariance of random matrix $\left[\mathbf{G}_{K_{n}}\right]$ under real orthogonal transformations.

\section{Monte Carlo numerical simulation of random matrix $\left[\mathbf{G}_{K_{n}}\right]$}

The following algebraic representation of positive-definite real random matrix $\left[\mathbf{G}_{K_{n}}\right]$ allows a procedure for the Monte Carlo numerical simulation of random matrix $\left[\mathbf{G}_{K_{n}}\right]$ to be defined. Random matrix $\left[\mathbf{G}_{K_{n}}\right]$ can be written as

$$
\left[\mathbf{G}_{K_{n}}\right]=\left[\mathbf{L}_{K_{n}}\right]^{T}\left[\mathbf{L}_{K_{n}}\right]
$$

in which $\left[\mathbf{L}_{K_{n}}\right]$ is an upper triangular random matrix with values in $M_{n}(\mathbb{R})$ such that:

(1) random variables $\left\{\left[\mathbf{L}_{K_{n}}\right]_{j j^{\prime}}, j \leq j^{\prime}\right\}$ are independent;

(2) for $j<j^{\prime}$, real-valued random variable $\left[\mathbf{L}_{K_{n}}\right]_{j j^{\prime}}$ can be written as $\left[\mathbf{L}_{K_{n}}\right]_{j j^{\prime}}=\sigma_{n} U_{j j^{\prime}}$ in which $\sigma_{n}=\delta_{K}(n+1)^{-1 / 2}$ and where $U_{j j^{\prime}}$ is a real-valued Gaussian random variable with zero mean and variance equal to 1 ; 
(3) for $j=j^{\prime}$, positive-valued random variable $\left[\mathbf{L}_{K_{n}}\right]_{j j}$ can be written as $\left[\mathbf{L}_{K_{n}}\right]_{j j}=\sigma_{n} \sqrt{2 V_{j}}$ in which $\sigma_{n}$ is defined above and where $V_{j}$ is a positive-valued gamma random variable for which the probability density function $p_{V_{j}}(v)$ with respect to $d v$ is written as

$$
p_{V_{j}}(v)=\mathbb{1}_{\mathbb{R}^{+}}(v) \frac{1}{\Gamma\left(\frac{n+1}{2 \delta_{K}^{2}}+\frac{1-j}{2}\right)} v^{\frac{n+1}{2 \delta_{K}^{2}}-\frac{1+j}{2}} e^{-v} .
$$

\subsection{PROBABILITY MODEL FOR A RANDOM MATRIX BELONGING TO THE GOE}

In this subsection, the random matrix $\left[\mathbf{K}_{n}^{\mathrm{GOE}}\right]$ with values in $\mathbb{M}_{n}^{S}(\mathbb{R})$ is constructed by using the Gaussian Orthogonal Ensemble (GOE) (concerning the GOE, see for instance reference [7] ). In order to perform the comparisons with the model summarized in Section 1.2, it is assumed that the mean value of random matrix $\left[\mathbf{K}_{n}^{\mathrm{GOE}}\right]$ is the positive-definite symmetric real matrix $\left[\underline{K}_{n}\right]$ defined by Eq. (1), that is to say

$$
E\left\{\left[\mathbf{K}_{n}^{\mathrm{GOE}}\right]\right\}=\left[\underline{K}_{n}\right] \in \mathbb{M}_{n}^{+}(\mathbb{R})
$$

\section{A. Normalization and dispersion parameter of random matrix $\left[\mathbf{K}_{n}^{\mathrm{GOE}}\right]$}

The developments of Section 1.2.A are used and consequently, random matrix $\left[\mathbf{K}_{n}^{\mathrm{GOE}}\right]$ can be written as

$$
\left[\mathbf{K}_{n}^{\mathrm{GOE}}\right]=\left[\underline{L}_{K_{n}}\right]^{T}\left[\mathbf{G}_{K_{n}}^{\mathrm{GOE}}\right]\left[\underline{L}_{K_{n}}\right],
$$

in which matrix $\left[\mathbf{G}_{K_{n}}^{\mathrm{GOE}}\right]$ is a random variable with values in $\mathbb{M}_{n}^{S}(\mathbb{R})$ such that

$$
\left[\underline{G}_{K}^{\mathrm{GOE}}\right]=E\left\{\left[\mathbf{G}_{K_{n}}^{\mathrm{GOE}}\right]\right\}=\left[I_{n}\right]
$$

In order to compare the two sets of random matrices in the same conditions, the dispersion parameter of random matrix $\left[\mathbf{K}_{n}^{\mathrm{GOE}}\right]$ is taken as parameter $\delta_{K}$ of random matrix $\left[\mathbf{K}_{n}\right]$, defined by Eq. (6). One then has

$$
\frac{E\left\{\left\|\left[\mathbf{G}_{K_{n}}^{\mathrm{GOE}}\right]-\left[\underline{G}_{K}^{\mathrm{GOE}}\right]\right\|_{F}^{2}\right\}}{\left\|\left[\underline{G}_{K_{n}}^{\mathrm{GOE}}\right]\right\|_{F}^{2}}=\delta_{K}^{2} \quad .
$$

It should be noted that $\left\|\left[\underline{G}_{K_{n}}^{\mathrm{GOE}}\right]\right\|_{F}^{2}=n$. As the mean value of a random matrix $\left[\mathbf{H}_{n}^{\mathrm{GOE}}\right]$ belonging to the GOE is such that $E\left\{\left[\mathbf{H}_{n}^{\mathrm{GOE}}\right]\right\}=[0]$, random matrix $\left[\mathbf{G}_{K_{n}}^{\mathrm{GOE}}\right]$ constructed with the GOE has to be written as

$$
\left[\mathbf{G}_{K_{n}}^{\mathrm{GOE}}\right]=\left[I_{n}\right]+\left[\mathbf{H}_{n}^{\mathrm{GOE}}\right]
$$


in which $\left[\mathbf{H}_{n}^{\mathrm{GOE}}\right]$ belongs to the GOE, that is to say, is a random matrix with values in $M_{n}^{S}(\mathbb{R})$. Consequently, random matrices $\left[\mathbf{H}_{n}^{\mathrm{GOE}}\right],\left[\mathbf{G}_{K_{n}}^{\mathrm{GOE}}\right]$ and $\left[\mathbf{K}_{n}^{\mathrm{GOE}}\right]$ are not positive matrices almost surely. Let $V_{j k}^{G_{K}^{\mathrm{GOE}}}$ be the variance of the random variable $\left[\mathbf{G}_{K_{n}}^{\mathrm{GOE}}\right]_{j k}$ such that

$$
V_{j k}^{G_{K}^{\mathrm{GOE}}}=E\left\{\left(\left[\mathbf{G}_{K_{n}}^{\mathrm{GOE}}\right]_{j k}-\left[\underline{G}_{K_{n}}^{\mathrm{GOE}}\right]_{j k}\right)^{2}\right\}=E\left\{\left(\left[\mathbf{H}_{n}^{\mathrm{GOE}}\right]_{j k}\right)^{2}\right\}
$$

Random matrix $\left[\mathbf{H}_{n}^{\mathrm{GOE}}\right]$ is constructed for that

$$
V_{j k}^{G_{K}^{\mathrm{GOE}}}=V_{j k}^{G_{K_{n}}}=\frac{\delta_{K}^{2}}{n+1}\left(1+\delta_{j k}\right) \quad, \quad j \text { and } k \in\{1, \ldots, n\}
$$

\section{B. Probability distribution and second-order moments of random matrix $\left[\mathbf{H}_{n}^{\mathrm{GOE}}\right]$}

With respect to the volume element $\widetilde{d} H_{n}$ defined by Eq. (7), the probability density function of random variable $\left[\mathbf{H}_{n}^{\mathrm{GOE}}\right]$ belonging to the GOE, such that its second-order moments are

$$
E\left\{\left[\mathbf{H}_{n}^{\mathrm{GOE}}\right]_{j k}\right\}=[0] \quad, \quad E\left\{\left(\left[\mathbf{H}_{n}^{\mathrm{GOE}}\right]_{j k}\right)^{2}\right\}=\frac{\delta_{K}^{2}}{n+1}\left(1+\delta_{j k}\right)
$$

is written as

$$
p_{\left[\mathbf{H}_{n}^{\mathrm{GOE}}\right]}\left(\left[H_{n}\right]\right)=C_{n} \times \exp \left\{-\frac{(n+1)}{4 \delta_{K}^{2}} \operatorname{tr}\left\{\left[H_{n}\right]^{2}\right\}\right\},
$$

where $C_{n}$ is the constant of normalization which can easily be calculated. Equation (27) shows that real-valued random variables $\left\{\left[\mathbf{H}_{n}^{\mathrm{GOE}}\right]_{j k}, j \leq k\right\}$ are mutually independent, second-order, centered and Gaussian.

\section{Additional properties of random matrix $\left[\mathbf{H}_{n}^{\mathrm{GOE}}\right]$}

The probability density function of random matrix $\left[\mathbf{H}_{n}^{\mathrm{GOE}}\right]$ defined by Eq. (27) shows that $\left[\mathbf{H}_{n}^{\mathrm{GOE}}\right]$ is invariant under real orthogonal transformations. Random matrix $\left[\mathbf{H}_{n}^{\mathrm{GOE}}\right]$ and consequently, random matrices $\left[\mathbf{G}_{K_{n}}^{\mathrm{GOE}}\right]$ and $\left[\mathbf{K}_{n}^{\mathrm{GOE}}\right]$ are with values in $\mathbb{M}_{n}^{S}(\mathbb{R})$ but not in $\mathbb{M}_{n}^{+}(\mathbb{R})$. Consequently, $\left[\mathbf{H}_{n}^{\mathrm{GOE}}\right],\left[\mathbf{G}_{K_{n}}^{\mathrm{GOE}}\right]$ and $\left[\mathbf{K}_{n}^{\mathrm{GOE}}\right]$ are not invertible almost surely and Eq. (15) does not hold neither for $\left[\mathbf{H}_{n}^{\mathrm{GOE}}\right]$ nor for $\left[\mathbf{G}_{K_{n}}^{\mathrm{GOE}}\right]$ or $\left[\mathbf{K}_{n}^{\mathrm{GOE}}\right]$.

\section{Monte Carlo numerical simulation of random matrix $\left[\mathbf{G}_{K_{n}}^{\mathrm{GOE}}\right]$}

As real-valued random variables $\left\{\left[\mathbf{H}_{n}^{\mathrm{GOE}}\right]_{j k}, j \leq k\right\}$ are mutually independent, second-order, centered and Gaussian with variances given by Eq. (26), then it is easy to perform a Monte Carlo numerical simulation of random matrix $\left[\mathbf{G}_{K_{n}}^{\mathrm{GOE}}\right]=\left[I_{n}\right]+\left[\mathbf{H}_{n}^{\mathrm{GOE}}\right]$. 


\section{STATISTICS OF THE RANDOM EIGENVALUES}

\subsection{INTRODUCING THE RANDOM GENERALIZED EIGENVALUE PROBLEMS}

With respect to the generalized coordinates $\underline{\mathbf{q}}=\left(\underline{q}_{1}, \ldots, \underline{q}_{n}\right) \in \mathbb{R}^{n}$ associated with the elastic modes corresponding to the $n$ lowest positive eigenfrequencies $0<\underline{\omega}_{1} \leq \ldots \leq \underline{\omega}_{n}$ of the fixed and stable mean dynamic system, the mean generalized eigenvalue problem is written (see Section 1.1) as

$$
\left.\left[\underline{K}_{n}\right] \underline{\mathbf{q}}=\underline{\lambda}^{[M} \underline{M}_{n}\right] \underline{\mathbf{q}} .
$$

The matrix $\left[\underline{M}_{n}\right] \in \mathbb{M}_{n}^{+}(\mathbb{R})$ is the mean generalized diagonal mass matrix for which the diagonal entries are the generalized masses of the elastic modes of the mean dynamic system. The matrix $\left[\underline{K}_{n}\right] \in \mathbb{M}_{n}^{+}(\mathbb{R})$ is the mean generalized diagonal stiffness matrix for which the diagonal entries are the $n$ first eigenvalues $0<\underline{\lambda}_{1} \leq \ldots \leq \underline{\lambda}_{n}$ of the mean dynamic system, such that $\underline{\lambda}_{j}=\underline{\omega}_{j}^{2}$. It should be noted that the mean generalized eigenvalue problem defined by Eq. (28) gives $n$-uncoupled equations for the mean dynamic systems due to the usual orthogonal properties of the elastic modes for a fixed (or a free) dynamic system. Let us assume that random uncertainties concern only the stiffness operator (the mass operator is certain for the reason given in the Introduction). The use of the non-parametric model of random uncertainties for this dynamic system consists in introducing (see Section 1.1) the random generalized eigenvalue problem associated with Eq. (28),

$$
\left[\mathbf{K}_{n}\right] \mathbf{Q}=\widetilde{\Lambda}\left[\underline{M}_{n}\right] \mathbf{Q}
$$

The probability model of the random matrix $\left[\mathbf{K}_{n}\right]$ with values in $\mathbb{M}_{n}^{+}(\mathbb{R})$ is defined in Section 1.2 and is such that $\left[\mathbf{K}_{n}\right]=\left[\underline{L}_{K_{n}}\right]^{T}\left[\mathbf{G}_{K_{n}}\right]\left[\underline{L}_{K_{n}}\right]$ in which $\left[\underline{L}_{K_{n}}\right]_{j k}=\underline{\lambda}_{j}^{1 / 2} \delta_{j k}$ and where the random matrix $\left[\mathbf{G}_{K_{n}}\right]$ with values in $\mathbf{M}_{n}^{+}(\mathbb{R})$ is such that

$$
E\left\{\left[\mathbf{G}_{K_{n}}\right]\right\}=\left[I_{n}\right] \quad, \quad V_{j k}^{G_{K_{n}}}=\frac{\delta_{K}^{2}}{n+1}\left(1+\delta_{j k}\right)
$$

The positive-valued random eigenvalues of Eq. (29) are denoted $0<\widetilde{\Lambda}_{1}, \ldots, \widetilde{\Lambda}_{n}$. It should be noted that the random generalized eigenvalue problem defined by Eq. (29) gives $n$-coupled random equations.

In order to compare the two ensembles of random matrices defined in Sections 1.2 and 1.3, a second random generalized eigenvalue problem is introduced by replacing random matrix $\left[\mathbf{K}_{n}\right]$ by the random matrix $\left[\mathbf{K}_{n}^{\mathrm{GOE}}\right]$ with values in $\mathbb{M}_{n}^{S}(\mathbb{R})$, defined in Section 1.3, which is such that $\left[\mathbf{K}_{n}^{\mathrm{GOE}}\right]=\left[\underline{L}_{K_{n}}\right]^{T}\left[\mathbf{G}_{K_{n}}^{\mathrm{GOE}}\right]\left[\underline{L}_{K_{n}}\right]$ where the random matrix $\left[\mathbf{G}_{K_{n}}^{\mathrm{GOE}}\right]$ with values in $\mathbb{M}_{n}^{+}(\mathbb{R})$ is such that

$$
E\left\{\left[\mathbf{G}_{K_{n}}^{\mathrm{GOE}}\right]\right\}=\left[I_{n}\right] \quad, \quad V_{j k}^{G_{K}^{\mathrm{GOE}}}=\frac{\delta_{K}^{2}}{n+1}\left(1+\delta_{j k}\right)
$$


This second random generalized eigenvalue problem is then written as

$$
\left[\mathbf{K}_{n}^{\mathrm{GOE}}\right] \mathbf{Q}^{\mathrm{GOE}}=\widetilde{\Lambda}^{\mathrm{GOE}}\left[\underline{M}_{n}\right] \mathbf{Q}^{\mathrm{GOE}}
$$

for which the real-valued random eigenvalues are denoted $\widetilde{\Lambda}_{1}^{\mathrm{GOE}}, \ldots, \widetilde{\Lambda}_{n}^{\mathrm{GOE}}$. Finally, the order statistics of random eigenvalues $0<\widetilde{\Lambda}_{1}, \ldots, \widetilde{\Lambda}_{n}$ and $\widetilde{\Lambda}_{1}^{\mathrm{GOE}}, \ldots, \widetilde{\Lambda}_{n}^{\mathrm{GOE}}$ are introduced,

$$
\begin{aligned}
& 0<\Lambda_{1} \leq \Lambda_{2} \leq \ldots \leq \Lambda_{n} \\
& \Lambda_{1}^{\mathrm{GOE}} \leq \Lambda_{2}^{\mathrm{GOE}} \leq \ldots \leq \Lambda_{n}^{\mathrm{GOE}}
\end{aligned}
$$

\subsection{PROBABILITY DENSITY FUNCTIONS AND SECOND-ORDER MOMENTS OF THE RANDOM EIGEN-}

\section{VALUES}

Let $p_{\Lambda_{j}}(\lambda) d \lambda$ and $p_{\Lambda_{j}^{\mathrm{GOE}}}(\lambda) d \lambda$ be the probability distributions of random eigenvalues $\Lambda_{j}$ and $\Lambda_{j}^{\mathrm{GOE}}$ respectively, corresponding to the order statistics defined by Eqs. (33) and (34). The mean values $m_{\Lambda_{j}}$ and $m_{\Lambda_{j}^{\mathrm{GOE}}}$, and the standard deviations $\sigma_{\Lambda_{j}}$ and $\sigma_{\Lambda_{j}^{\mathrm{GOE}}}$ of the random eigenvalues $\Lambda_{j}$ and $\Lambda_{j}^{\mathrm{GOE}}$ are such that

$$
\begin{aligned}
& m_{\Lambda_{j}}=E\left\{\Lambda_{j}\right\} \quad, \quad m_{\Lambda_{j}^{\mathrm{GOE}}}=E\left\{\Lambda_{j}^{\mathrm{GOE}}\right\}, \\
& \sigma_{\Lambda_{j}}^{2}=E\left\{\Lambda_{j}^{2}\right\}-m_{\Lambda_{j}}^{2} \quad, \quad \sigma_{\Lambda_{j}^{\mathrm{GOE}}}^{2}=E\left\{\left(\Lambda_{j}^{\mathrm{GOE}}\right)^{2}\right\}-m_{\Lambda_{j}^{\mathrm{GOE}}}^{2}
\end{aligned}
$$

in which the moments of order $\nu \geq 1$ are defined by

$$
E\left\{\Lambda_{j}^{\nu}\right\}=\int_{0}^{+\infty} \lambda^{\nu} p_{\Lambda_{j}}(\lambda) d \lambda \quad, \quad E\left\{\left(\Lambda_{j}^{\mathrm{GOE}}\right)^{\nu}\right\}=\int_{-\infty}^{+\infty} \lambda^{\nu} p_{\Lambda_{j}^{\mathrm{GOE}}}(\lambda) d \lambda .
$$

For the general case considered, probability density functions $p_{\Lambda_{j}}(\lambda)$ and $p_{\Lambda_{j}^{\mathrm{GOE}}}(\lambda)$ and the second-order moments cannot be explicitly constructed. This is the reason why an approximation of these quantities will be constructed by using the Monte Carlo numerical simulation.

\subsection{PROBABILITY DENSITY FUNCTIONS AND SECOND-ORDER MOMENTS OF THE RANDOM NOR-}

\section{MALIZED SPACINGS BETWEEN TWO CONSECUTIVE RANDOM EIGENVALUES}

The random spacing $\Delta_{j}$ (or $\Delta_{j}^{\mathrm{GOE}}$ ) between the two consecutive random eigenvalues $\Lambda_{j}$ and $\Lambda_{j+1}$ (or $\Lambda_{j}^{\mathrm{GOE}}$ and $\Lambda_{j+1}^{\mathrm{GOE}}$ ) of the order statistics is defined by

$$
\Delta_{j}=\Lambda_{j+1}-\Lambda_{j} \quad, \quad\left(\text { or } \quad \Delta_{j}^{\mathrm{GOE}}=\Lambda_{j+1}^{\mathrm{GOE}}-\Lambda_{j}^{\mathrm{GOE}}\right) \quad, \quad j \in\{1, \ldots, n-1\} .
$$

The mean value $m_{\Delta_{j}}$ (or $m_{\Delta_{j}^{\mathrm{GOE}}}$ ) of random variable $\Delta_{j}$ (or $\Delta_{j}^{\mathrm{GOE}}$ ) is defined by

$$
m_{\Delta_{j}}=E\left\{\Delta_{j}\right\} \quad, \quad\left(\text { or } \quad m_{\Delta_{j}^{\mathrm{GOE}}}=E\left\{\Delta_{j}^{\mathrm{GOE}}\right\}\right) \quad, \quad j \in\{1, \ldots, n-1\}
$$


In Section 2.4, one will see that $m_{\Delta_{j}}$ ( or $m_{\Delta_{j}^{\mathrm{GOE}}}$ ) depends on $j$. As usually, the random normalized spacing $S_{j}$ (or $S_{j}^{\mathrm{GOE}}$ ) can be introduced and is defined by

$$
S_{j}=\frac{\Delta_{j}}{m_{\Delta_{j}}} \quad, \quad\left(\text { or } \quad S_{j}^{\mathrm{GOE}}=\frac{\Delta_{j}^{\mathrm{GOE}}}{m_{\Delta_{j}^{\mathrm{GOE}}}}\right) \quad, \quad j \in\{1, \ldots, n-1\} .
$$

It can easily be seen that the mean value $m_{S_{j}}=E\left\{S_{j}\right\}$ (or $m_{S_{j}^{\mathrm{GOE}}}=E\left\{S_{j}^{\mathrm{GOE}}\right\}$ ) of random variables $S_{j}$ (or $S_{j}^{\mathrm{GOE}}$ ) is independent of $j$ and is such that

$$
m_{S_{j}}=1 \quad, \quad\left(\text { or } \quad m_{S_{j}^{\mathrm{GOE}}}=1\right) \quad, \quad j \in\{1, \ldots, n-1\} \quad .
$$

For $j$ fixed in $\{1,2, \ldots, n-1\}$, let $p_{S_{j}}(s)$ (or $p_{S_{j}^{\operatorname{GOE}}}(s)$ ) be the probability density function with respect to $d s$ of positive-valued random variable $S_{j}$ (or $S_{j}^{\mathrm{GOE}}$ ), . Let $\sigma_{S_{j}}$ (or $\sigma_{S_{j}^{\mathrm{GOE}}}$ ) be the standard deviation of random variable $S_{j}\left(\right.$ or $S_{j}^{\mathrm{GOE}}$ ), such that

$$
\begin{gathered}
\sigma_{S_{j}}^{2}=E\left\{S_{j}^{2}\right\}-1 \quad, \quad\left(\text { or } \quad \sigma_{S_{j}^{\mathrm{GOE}}}^{2}=E\left\{\left(S_{j}^{\mathrm{GOE}}\right)^{2}\right\}-1\right), \\
E\left\{S_{j}^{2}\right\}=\int_{0}^{+\infty} s^{2} p_{S_{j}}(s) d s \quad, \quad\left(\text { or } \quad E\left\{\left(S_{j}^{\mathrm{GOE}}\right)^{2}\right\}=\int_{0}^{+\infty} s^{2} p_{S_{j}^{\mathrm{GOE}}}(s) d s .\right.
\end{gathered}
$$

Probability density function $p_{S_{j}}(s)$ ( or $p_{S_{j}^{\mathrm{GOE}}}(s)$ ), and consequently, $\sigma_{S_{j}}$ (or $\sigma_{S_{j}^{\mathrm{GOE}}}$ ) depends, a priori, on $j$ (this statement will be verified in Section 2.4). Nevertheless, one will see in Section 2.4, that function $j \mapsto \sigma_{S_{j}}$ (or $j \mapsto \sigma_{S_{j}^{\mathrm{GOE}}}$ depends weakly on $j$. Consequently, following the usual approach (see for instance reference [7]), one introduces the positive-valued spacing random variable $S$ (or $S^{\mathrm{GOE}}$ ) such that, for all $\theta$ fixed in $\mathcal{A}, S_{1}(\theta), \ldots, S_{n-1}(\theta)$ (or $\left.S_{1}^{\mathrm{GOE}}(\theta), \ldots, S_{n-1}^{\mathrm{GOE}}(\theta)\right)$ are $n-1$ independent realizations of random variable $S$ (or $S^{\mathrm{GOE}}$ ). It should be noted that this construction corresponds to an approximation. The probability density function $p_{S}(s)$ (or $p_{S^{\operatorname{GOE}}}(s)$ ) with respect to $d s$ of positive-valued random variable $S$ (or $S^{\mathrm{GOE}}$ ) are usually called the spacing probability density function. Concerning the GOE, the Wigner surmise for the spacing probability density function consists in writting (see [7]) that $p_{S^{\mathrm{GOE}}}(s) \simeq p_{W}(s)$ in which

$$
p_{W}(s)=\mathbb{1}_{\mathbb{R}^{+}}(s) \frac{\pi}{2} s e^{-\frac{\pi}{4} s^{2}} .
$$

However, a more general probability density function than $p_{W}(s)$ was introduced by Brody [35] for fitting spacing probability density functions. This probability density function, denoted $p_{B}(s)$, is written as

$$
p_{B}(s)=\mathbb{1}_{\mathbb{R}^{+}}(s) a s^{\alpha-1} e^{-b s^{\alpha}},
$$

in which $\alpha>0, a>0$ and $b>0$ are such that $\int_{0}^{+\infty} p_{B}(s) d s=1$ and $\int_{0}^{+\infty} s p_{B}(s) d s=1$. One then gets $a=\alpha b$ and $b=\{\Gamma(1+1 / \alpha)\}^{\alpha}$. Taking $\alpha=2$ yields $p_{B}(s)=p_{W}(s)$. In Section 2.4 , it is proved that $p_{B}(s)$ with $\alpha<2$ is a better approximation of $p_{S}(s)$ and $p_{S^{\mathrm{GOE}}}(s)$ than $p_{W}(s)$. 
Below, the ensemble of random matrices defined in Section 1.2 will be called the "positivedefinite" ensemble. One considers the mean reduced system with dimension $n=30$, such that, for all $\alpha$ and $\beta$ in $\{1, \ldots, 30\}$,

$$
\left[\underline{M}_{n}\right]_{\alpha \beta}=\delta_{\alpha \beta},\left[\underline{K}_{n}\right]_{\alpha \beta}=\underline{k}_{1} \int_{-1}^{+1} \underline{\varphi}_{\alpha}^{\prime \prime}(x) \underline{\varphi}_{\beta}^{\prime \prime}(x) d x+\underline{k}_{2} \int_{-1}^{+1} \underline{\varphi}_{\alpha}(x) \underline{\varphi}_{\beta}(x) d x,
$$

in which $\underline{\varphi}_{\alpha}(x)=\sin (\pi \alpha(1+x) / 2)$ and $\underline{\varphi}_{\alpha}^{\prime \prime}$ is the second derivative of $\underline{\varphi}_{\alpha}$ with respect to $x$. This model corresponds to an Euler beam in bending mode, with length 2, simply supported at its ends, attached to a continuous elastic support along its length, for which the elastic bending modes $\underline{\varphi}_{\alpha}$ are associated with the 30 lowest eigenfrequencies $\underline{\omega}_{\alpha}$ such that

$$
\underline{\omega}_{\alpha}^{2}=\underline{\lambda}_{\alpha}=\underline{k}_{1}\left(\frac{\alpha \pi}{2}\right)^{4}+\underline{k}_{2}
$$

For all the numerical examples considered in this paper, one takes $\underline{k}_{1}=0.9999987$ and $\underline{k}_{2}=2.0278508 \times 10^{-7}$. Consequently, one has $1 \leq \underline{\lambda}_{\alpha} \leq 2$ with $\underline{\lambda}_{1}=1, \underline{\lambda}_{2}=1.000185$, $\ldots, \underline{\lambda}_{29}=1.873186, \underline{\lambda}_{30}=2$. As explained in Section 2.2, the Monte Carlo numerical simulation [36,37] and the usual mathematical statistics are used to estimate $m_{\Lambda_{j}}, m_{\Lambda_{j}^{\mathrm{GOE}}}, m_{\Delta_{j}}$, $m_{\Delta_{j}^{\mathrm{GOE}}}, \sigma_{\Lambda_{j}}, \sigma_{\Lambda_{j}^{\mathrm{GOE}}}, \sigma_{S_{j}}, \sigma_{S_{j}^{\mathrm{GOE}}}$ and probability density functions $p_{\Lambda_{j}}, p_{\Lambda_{j}^{\mathrm{GOE}}}, p_{S_{j}}(s), p_{S_{j}^{\mathrm{GOE}}}(s)$, $p_{S}(s), p_{S^{\mathrm{GOE}}}(s)$, defined in Sections 2.2 and 2.3. Figures 1 to 8 (left and right) display the results corresponding to the use of $10^{6}$ realizations in the computation. There are two groups of figures. Figures 1 to 4 correspond to $\delta_{K}=0.25$ (weak value of the dispersion parameter) and Figures 5 to 8 correspond to $\delta_{K}=0.50$ (strong value of the dispersion parameter)

\section{A. Results for $\delta_{K}=0.25$}

Figures 1 compare the graph of function $j \mapsto m_{\Lambda_{j}}$ with the graph of function $j \mapsto m_{\Lambda_{j}^{\mathrm{GOE}}}$ (Figure 1 on the left) and, $j \mapsto \sigma_{\Lambda_{j}}$ with $j \mapsto \sigma_{\Lambda_{j}^{\mathrm{GOE}}}$ (Figure 1 on the right), the functions being defined on the set $\{1, \ldots, 30\}$. For this weak value of dispersion parameter $\delta_{K}$, it can be seen that the second-order moments of the random eigenvalues (order statistics) are similar for the "positive-definite" ensemble and for the GOE. Figure 2 on the left displays the 30 graphs of probability density functions (pdf) $\left\{\lambda \mapsto p_{\Lambda_{j}^{\mathrm{GOE}}}(\lambda), j=1, \ldots, 30\right\}$ for the GOE. Figure 2 on the right displays the 30 graphs of $\operatorname{pdf}\left\{\lambda \mapsto p_{\Lambda_{j}}(\lambda), j=1, \ldots, 30\right\}$ for the "positive-definite" ensemble. For this weak value of $\delta_{K}$, the graphs are similar for the two ensembles of random matrices. Figures 3 compare the graph of function $j \mapsto m_{\Delta_{j}}$ with the graph of function $j \mapsto m_{\Delta_{j}^{\mathrm{GOE}}}$ (Figure 3 on the left) and,$j \mapsto \sigma_{S_{j}}$ with $j \mapsto \sigma_{S_{j}^{\mathrm{GOE}}}$ (Figure 3 on the right). The results for the second-order moments of the random spacings are similar for 
the "positive-definite" ensemble and for the GOE. However, it should be noted (see Figure 3 on the right) that $\sigma_{S_{j}}$ and $\sigma_{S_{j}^{\mathrm{GOE}}}$ depend lightly on $j$, as explained in Section 2.3. Figure 4-a on the left shows the 30 graphs of pdf $\left\{s \mapsto \log _{10}\left(p_{S_{j}}(s)\right), j=1, \ldots, 30\right\}$ compared with the Wigner pdf $s \mapsto \log _{10}\left(p_{W}(s)\right)$. This figure shows that, for $s>2.5$, the Wigner pdf does not fit well pdf $\left\{p_{S_{j}}, j=1, \ldots, 30\right\}$ in mean. Figure 4 -a on the right shows the graph of pdf $s \mapsto \log _{10}\left(p_{S}(s)\right)$ (irregular thick solid line), in which $p_{S}(s)$ is estimated as explained in Section 2.3, compared with the Wigner pdf $s \mapsto \log _{10}\left(p_{W}(s)\right)$ (dashed line). For $s>2.5$, it can be seen that $p_{W}(s)$ does not fit well $p_{S}(s)$. Consequently, a Brody pdf $s \mapsto \log _{10}\left(p_{B}(s)\right)$ has been fitted with $\alpha=1.93$ (thin solid line). Figures 4-b (left and right) are similar to Figures 4-a, but correspond to the GOE instead of the "positive-definite" ensemble. Figure 4-b on the left show pdf $\left\{s \mapsto \log _{10}\left(p_{S_{j}^{\mathrm{GOE}}}(s)\right), j=1, \ldots, 30\right\}$ and $s \mapsto \log _{10}\left(p_{W}(s)\right)$. Figure 4-b on the right shows the graph of pdf $s \mapsto \log _{10}\left(p_{S^{\mathrm{GOE}}}(s)\right)$ (irregular thick solid line), $s \mapsto \log _{10}\left(p_{W}(s)\right)$ (dashed line) and $s \mapsto \log _{10}\left(p_{B}(s)\right)$ fitted with $\alpha=1.93$ (thin solid line). The analysis of Figures 4-a and 4-b show that, for the two ensembles of random matrices, the spacing probability density functions are similar. However, their assymptotic behavior at infinity does not follow the Wigner pdf but are better fitted with the Brody pdf with $\alpha=1.93$.

\section{B. Results for $\delta_{K}=0.50$}

Figures 5 to 8 correspond to Figures 1 to 4 for the strong value of dispersion parameter $\delta_{K}$ instead of its small value. Figures 5 compare the graphs of functions $j \mapsto m_{\Lambda_{j}}$ with $j \mapsto m_{\Lambda_{j}^{\mathrm{GOE}}}$ (Figure 5 on the left) and, compare the graphs of functions $j \mapsto \sigma_{\Lambda_{j}}$ with $j \mapsto \sigma_{\Lambda_{j}^{\mathrm{GOE}}}$ (Figure 5 on the right). For this strong value of dispersion parameter $\delta_{K}$, the second-order moments of the random eigenvalues (order statistics) are different for the "positive-definite" ensemble and for the GOE, especially, for the standard deviations. For the "positive-definite" ensemble, $j \mapsto \sigma_{\Lambda_{j}}$ is a monotonic increasing function, that is not the case for function $j \mapsto \sigma_{\Lambda_{j}^{\mathrm{G}}}$ corresponding to the GOE. This point constitutes an important difference between the two ensembles. It should be noted that, in dynamic systems, the random uncertainties increase with frequency and consequently, the standard deviation $\sigma_{\Lambda_{j}}$ has to increase with $j$ from $j=1$. This is the case for the "positive-definite" ensemble but not the case for the GOE, i.e for $\sigma_{\Lambda_{j}^{\mathrm{GOE}}}$. Figure 6 on the left displays the 30 graphs of pdf $\left\{\lambda \mapsto p_{\Lambda_{j}^{\mathrm{GOE}}}(\lambda), j=1, \ldots, 30\right\}$ for the GOE. Figure 6 on the right displays the 30 graphs of $\operatorname{pdf}\left\{\lambda \mapsto p_{\Lambda_{j}}(\lambda), j=1, \ldots, 30\right\}$ for the "positive-definite" ensemble. The "positive-definite" ensemble yields results very different from the GOE. Those given by the GOE are not good: in Figure 6 on the left, it can easily be seen that $p_{\Lambda_{1}^{\mathrm{GOE}}}(\lambda)$ is not equal to zero for $\lambda<0$. This means that random eigenvalue $\Lambda_{1}^{\mathrm{GOE}}$ (that is to say the fundamental eigenfrequency of the dynamic system with random uncertainties) 
is not positive almost surely, that is not admissible for a stable dynamic system. In opposite, the "positive-definite" ensemble gives good results. Figures 7 compare the graphs of functions $j \mapsto m_{\Delta_{j}}$ with $j \mapsto m_{\Delta_{j}^{\mathrm{GOE}}}$ (Figure 7 on the left) and,$j \mapsto \sigma_{S_{j}}$ with $j \mapsto \sigma_{S_{j}^{\mathrm{GOE}}}$ (Figure 7 on the right). Concerning the mean values (Figure 7 on the left), there is a significant difference between the two ensembles while the difference is small for standard deviations $\sigma_{S_{j}}$ and $\sigma_{S_{j}^{\mathrm{GOE}}}$ which depend lightly on $j$. Figures 8 -a and 8-b, which correspond to the strong value of dispersion parameter $\delta_{K}$, are very similar to Figures 4-a and 4-b which correspond to the small value of the dispersion parameter. Figures 8-a correspond to the "positive-definite" ensemble and Figures 8-b to the GOE. For this strong value of the dispersion parameter, the Brody pdf is well fitted with $\alpha=1.91$ instead of $\alpha=1.93$ for the small value.

\section{NON-PARAMETRIC MODEL OF RANDOM UNCERTAINTIES IN VIBRATION ANALYSIS}

\subsection{DEFINITION OF THE MEAN REDUCED MODEL OF THE DYNAMIC SYSTEM}

One considers a fixed stable linear mean dynamic system for which the Fourier transform $\underline{\mathbf{u}}(\mathbf{x}, \omega)$ with respect to $t$ of the vector-valued displacement field $\underline{\mathbf{u}}(\mathbf{x}, t)$, is defined on a bounded domain $\underline{\Omega} \subset \mathbb{R}^{d}$ with $d \geq 1$, equipped with the measure denoted $d \mathbf{x}$ and such that $|\underline{\Omega}|=\int_{\underline{\Omega}} d \mathbf{x}$ is the "volume" of domain $\underline{\Omega}$. For all $\omega$ belonging to the frequency band of analysis $\left[0, \omega_{\max }\right]$ with $\omega_{\max }>0$, the mean reduced model of dimension $n \geq 1$ of this mean dynamic system is obtained by using the usual mode-superposition method. The approximation $\underline{\mathbf{u}}_{n}(\mathbf{x}, \omega)$ of $\underline{\mathbf{u}}(\mathbf{x}, \omega)$ with dimension $n$ is then written as

$$
\begin{gathered}
\underline{\mathbf{u}}_{n}(\mathbf{x}, \omega)=\sum_{\alpha=1}^{n} \underline{q}_{\alpha}(\omega) \underline{\varphi}_{\alpha}(\mathbf{x}) \quad, \quad \mathbf{x} \in \underline{\Omega}, \\
\left(-\omega^{2}\left[\underline{M}_{n}\right]+i \omega\left[\underline{D}_{n}\right]+\left[\underline{K}_{n}\right]\right) \underline{\mathbf{q}}(\omega)=\mathbf{f}(\omega),
\end{gathered}
$$

in which $\underline{\varphi}_{1}, \ldots, \underline{\varphi}_{n}$ are the elastic modes corresponding to the $n$ lowest eigenfrequencies $0<\underline{\omega}_{1} \leq \underline{\omega}_{2} \leq \ldots \leq \underline{\omega}_{n}$ of the mean dynamic system, $\underline{\mathbf{q}}(\omega)=\left(\underline{q}_{1}(\omega), \ldots, \underline{q}_{n}(\omega)\right) \in \mathbb{C}^{n}$ is the complex vector of the generalized coordinates, $\mathbf{f}(\omega)=\left(f_{1}(\omega), \ldots, f_{n}(\omega)\right) \in \mathbb{C}^{n}$ is the complex vector of the generalized external forces, $\left[\underline{M}_{n}\right],\left[\underline{D}_{n}\right]$ and $\left[\underline{K}_{n}\right]$ belong to $\mathbb{M}_{n}^{+}(\mathbb{R})$ and represent the generalized diagonal mass matrix, the generalized full damping matrix and the generalized diagonal stiffness matrix, respectively. It is assumed that the mass density of the mean dynamic system is a constant equal to 1 , that $\int_{\underline{\Omega}} \underline{\varphi}_{\alpha}(\mathbf{x}) \cdot \underline{\varphi}_{\beta}(\mathbf{x}) d \mathbf{x}=\delta_{\alpha \beta}$ and that the generalized damping matrix is a diagonal matrix, such that

$$
\left[\underline{M}_{n}\right]=\delta_{\alpha \beta} \quad, \quad\left[\underline{D}_{n}\right]=2 \underline{\xi} \omega_{\text {ref }} \delta_{\alpha \beta} \quad, \quad\left[\underline{K}_{n}\right]=\underline{\omega}_{\alpha}^{2} \delta_{\alpha \beta} \quad,
$$


in which $\underline{\xi}>0$ and $\omega_{\text {ref }}>0$ are given positive constants. From Eq. (50), it can be deduced that, for the mean dynamic system, the critical damping rates $\underline{\xi}_{1}, \ldots, \underline{\xi}_{n}$ of elastic modes $\underline{\varphi}_{1}, \ldots, \underline{\varphi}_{n}$ are given by $\underline{\xi}_{\alpha}=\underline{\xi} \omega_{\text {ref }} / \underline{\omega}_{\alpha}$. It should be noted that for the numerical examples presented in this paper, the given mean value of the critical damping rates of the elastic modes whose eigenfrequencies are inside the frequency band of analysis, is taken as 0.001. In this case, the use of Eq. (50) yields as the minimum value of the critical damping rates $\underline{\xi}_{\alpha}$, the value 0.0009 , and as the maximum value, the value 0.0012. Consequently, for the mean dynamic system, the use of a simple Rayleigh damping is sufficient in taking into account the objectives of this paper. As observation of the mean dynamic system, one introduces the positive-valued function $\omega \mapsto \underline{e}_{n}(\omega)$ such that

$$
\underline{e}_{n}(\omega)=\left\|\omega^{2}\left[\widehat{h}_{n}(\omega)\right]\right\|_{F}
$$

in which $\left[\widehat{h}_{n}(\omega)\right]=\left(-\omega^{2}\left[\underline{M}_{n}\right]+i \omega\left[\underline{D}_{n}\right]+\left[\underline{K}_{n}\right]\right)^{-1}$ is the generalized frequency response function of the mean dynamic system and $\|[A]\|_{F}=\left(\operatorname{tr}\left\{[A][A]^{*}\right\}\right)^{1 / 2}$ with $\left.[A]^{*}=\overline{[A}\right]^{T}$.

\subsection{NON-PARAMETRIC MODEL OF RANDOM UNCERTAINTIES}

The non-parametric model of random uncertainties is introduced as explained in Section 1.1. For preserving the coherence with Section 2.1, it is assumed that only the stiffness operator is uncertain. Consequently, the use of the "positive-definite" ensemble for the non-parametric modelling of random uncertainties leads us to the following random generalized frequency response function of the random dynamic system,

$$
\left[\mathbf{H}_{n}(\omega)\right]=\left(-\omega^{2}\left[\underline{M}_{n}\right]+i \omega\left[\underline{D}_{n}\right]+\left[\mathbf{K}_{n}\right]\right)^{-1},
$$

in which the probability model of random matrix $\left[\mathbf{K}_{n}\right]$ is defined in Section 1.2. The random observation associated with Eq. (51), is the positive-valued random variable $\mathcal{E}_{n}(\omega)$ defined by

$$
\mathcal{E}_{n}(\omega)=\left\|\omega^{2}\left[\mathbf{H}_{n}(\omega)\right]\right\|_{F}
$$

If the generalized stiffness matrix is modelled by the GOE, then $\left[\mathbf{K}_{n}\right]$ is replaced by $\left[\mathbf{K}_{n}^{\mathrm{GOE}}\right]$ for which the probability model is defined in Section 1.3. In this case, $\mathcal{E}_{n}(\omega)$ is denoted $\mathcal{E}_{n}^{\mathrm{GOE}}(\omega)$.

\subsection{COMPARISON OF THE TWO ENSEMBLES OF RANDOM MATRICES}

The mean reduced model of the dynamic system is defined in Sections 2.4 and 3.1 with $\underline{\xi}=0.01$ and $\omega_{\text {ref }}=2 \pi \times 0.02 \mathrm{rad} / \mathrm{s}$. The frequency band of analysis is such that $\omega_{\max }=2 \pi \times$ 
$0.22 \mathrm{rad} / \mathrm{s}$. The value of the dispersion parameter is $\delta_{K}=0.50$. The Monte Carlo numerical simulation method is carried out with $n_{s}=40000$ realizations, denoted $\theta_{1}, \ldots \theta_{n_{s}}$, for which the realizations $\omega \mapsto \mathcal{E}_{n}\left(\omega ; \theta_{1}\right), \ldots, \omega \mapsto \mathcal{E}_{n}\left(\omega ; \theta_{n_{s}}\right)$ are numerically calculated for the two ensembles of random matrices, with a sampling frequency step $\Delta \omega=\omega_{\max } / 300$. For $\omega$ fixed in $\left[0, \omega_{\max }\right]$, the mean values $E\left\{\mathcal{E}_{n}(\omega)\right\}$ and $E\left\{\mathcal{E}_{n}^{\mathrm{GOE}}(\omega)\right\}$, and the standard deviations $\sigma_{\mathcal{E}_{n}}(\omega)$ and $\sigma_{\mathcal{E}_{n}^{\mathrm{GOE}}}(\omega)$, of random variables $\mathcal{E}_{n}(\omega)$ and $\mathcal{E}_{n}^{\mathrm{GOE}}(\omega)$ respectively, are usually estimated. For the comparisons, one defines the functions $\nu \mapsto \mathrm{dB}(\nu)$ and $\nu \mapsto \mathrm{dB}^{\mathrm{GOE}}(\nu)$ such that

$$
\mathrm{dB}(\nu)=\log _{10}\left(E\left\{\mathcal{E}_{n}(2 \pi \nu)\right\}\right) \quad, \quad \mathrm{dB}^{\mathrm{GOE}}(\nu)=\log _{10}\left(E\left\{\mathcal{E}_{n}^{\mathrm{GOE}}(2 \pi \nu)\right\}\right) .
$$

Finally, for the "positive-definite" ensemble and for the GOE, for all $\nu$ fixed in $\left[0, \omega_{\max } / 2 \pi\right]$, the extreme value statistics associated with realizations $\theta_{1}, \ldots, \theta_{n_{s}}$ are defined by

$$
\begin{aligned}
& \mathrm{dB}_{\max }(\nu)=\log _{10}\left\{\max _{k} \mathcal{E}_{n}\left(2 \pi \nu ; \theta_{k}\right)\right\} \quad, \quad \mathrm{dB}_{\min }(\nu)=\log _{10}\left\{\min _{k} \mathcal{E}_{n}\left(2 \pi \nu ; \theta_{k}\right)\right\}, \\
& \mathrm{dB}_{\max }^{\mathrm{GOE}}(\nu)=\log _{10}\left\{\max _{k} \mathcal{E}_{n}^{\mathrm{GOE}}\left(2 \pi \nu ; \theta_{k}\right)\right\}, \mathrm{dB}_{\min }^{\mathrm{GOE}}(\nu)=\log _{10}\left\{\min _{k} \mathcal{E}_{n}^{\mathrm{GOE}}\left(2 \pi \nu ; \theta_{k}\right)\right\} .
\end{aligned}
$$

Figures 9 and 10 are relative to the frequency band $[0,0.22] \mathrm{Hz}$. Figure 9 on the left displays (1) the response $\nu \mapsto \log _{10} \underline{e}_{n}(2 \pi \nu)$ of the mean dynamic system (dashed line), (2) the graphs of functions $\nu \mapsto \mathrm{dB}(\nu)$ (thick solid line) and $\nu \mapsto \mathrm{dB}^{\mathrm{GOE}}(\nu)$ (thin solid line). Figure 9 on the right displays the graphs of functions $\nu \mapsto \sigma_{\mathcal{E}_{n}}(2 \pi \nu)$ (thick solid line) and $\nu \mapsto \sigma_{\mathcal{E}_{n}^{\mathrm{GOE}}}(2 \pi \nu)$ (thin solid line). Figures 9 show an important difference between the "positive-definite" ensemble and the GOE. As proved in Section 2.4.B, for the GOE, the first random eigenvalues (the lowest eigenvalues of the order statistics) have a larger standard deviation than for the "positive-definite" ensemble and their probability distributions are different. This is the reason why the mean value and the standard deviation of the random responses are very different in the frequency band $[0,0.1] \mathrm{Hz}$ for the two ensembles of random matrices. These differences can also be seen in Figure 10 which shows (1) for the "positive-definite" ensemble, the graphs of functions $\nu \mapsto \mathrm{dB}(\nu)$ (thick dashed line), $\nu \mapsto \mathrm{dB}_{\max }(\nu)$ (upper thick solid line), $\nu \mapsto \mathrm{dB}_{\min }(\nu)$ (lower thick solid line), (2) for the GOE, the graphs of functions $\nu \mapsto \mathrm{dB}^{\mathrm{GOE}}(\nu)$ (thin dashed line), $\nu \mapsto \mathrm{dB}_{\max }^{\mathrm{GOE}}(\nu)$ (upper thin solid line), $\nu \mapsto \mathrm{dB}_{\min }^{\mathrm{GOE}}(\nu)$ (lower thin solid line).

\section{A VALIDATION POINT FOR THE NON-PARAMETRIC MODEL OF RANDOM UNCERTAINTIES IN VIBRATION ANALYSIS}

\subsection{SETTING THE PROBLEM}

As explained in Section 1.1 of the introduction, the non-parametric model of random uncertainties in vibration analysis has been introduced to replace the usual parametric model for 
complex dynamic systems when the number of uncertain local parameters is large and above all, to take into account the model uncertainties which cannot be modelled with the parametric models. Nevertheless, as Section 3 shows that the results given by the two ensembles of random matrices are very different, it is interesting to analyze a simple dynamic system with random uncertainties which can easily be modelled by using the usual parametric approach, in order to conclude if the use of the "positive-definite" ensemble is better than the use of the GOE for the non-parametric model of random uncertainties in low-frequency vibration analysis. Consequently, one considers the dynamic system with parametric random uncertainties for which the associated mean dynamic system is defined in Section 2.4 and which is used in Section 3.

\subsection{DEFINING THE DYNAMIC SYSTEM WITH PARAMETRIC RANDOM UNCERTAINTIES}

One considers a dynamic system with parametric random uncertainties on the stiffness operator, for which the mean dynamic system is defined in Sections 2.4 and 3.1. In the frequency domain, the weak formulation of the corresponding boundary value problem is written as

$$
\begin{gathered}
-\omega^{2} \int_{-1}^{+1} U(x, \omega) v(x) d x+2 i \omega \underline{\xi} \omega_{\text {ref }} \int_{-1}^{+1} U(x, \omega) v(x) d x+\underline{k}_{1} \int_{-1}^{+1} Y(x) U^{\prime \prime}(x, \omega) v^{\prime \prime}(x) d x \\
\quad+\underline{k}_{2} \int_{-1}^{+1} T(x) U(x, \omega) v(x) d x=\int_{-1}^{+1} g(x, \omega) v(x) d x
\end{gathered}
$$

in which $v^{\prime \prime}$ is the second derivative of $v$ with respect to $x$ and where the test function $v$ belongs to the admissible function space constituted of the "sufficiently differentiable" real-valued functions $v$ defined on $\underline{\Omega}=]-1,+1[$ and such that $v(-1)=v(+1)=0$ and $v^{\prime \prime}(-1)=v^{\prime \prime}(+1)=0$. The external excitation is represented by the complex-valued force field $x \mapsto g(x, \omega)$ define on $\underline{\Omega}$. In Eq. (57), $\underline{\xi}$ ans $\omega_{\text {ref }}$ are defined in Section 3.3 and, $\underline{k}_{1}$ and $\underline{k}_{2}$ are defined in Section 2.4. Parameters $Y(x)$ and $T(x)$ are second-order stochastic processes indexed by $\underline{\Omega}$ with values in $\mathbb{R}^{+}$, statistically independent, such that

$$
E\{Y(x)\}=1 \quad, \quad E\{T(x)\}=1 \quad, \quad \forall x \in \underline{\Omega} .
$$

For $x$ fixed in $\underline{\Omega}, Y(x)$ and $T(x)$ are written as

$$
Y(x)=\frac{1}{m_{Y}} \sum_{j=1}^{m_{Y}} Z_{j}^{Y}(x)^{2} \quad, \quad T(x)=\frac{1}{m_{T}} \sum_{j=1}^{m_{T}} Z_{j}^{T}(x)^{2},
$$

in which $m_{Y} \geq 1$ and $m_{T} \geq 1$ are two finite positive integers and where $Z_{1}^{Y}, \ldots, Z_{m_{Y}}^{Y}$, $Z_{1}^{T}, \ldots, Z_{m_{T}}^{T}$ are $m_{Y}+m_{T}$ independent copies of a stochastic process $Z_{b}$ defined as follows. Stochastic process $\left\{Z_{b}(x), x \in \mathbb{R}\right\}$ is indexed by $\mathbb{R}$, with values in $\mathbb{R}$, second-order, centered, Gaussian and stationary, such that

$$
E\left\{Z_{b}(x)\right\}=0 \quad, \quad E\left\{Z_{b}(x)^{2}\right\}=1
$$


Let $S_{Z_{b}}(k)$ be its power spectral density function defined on $\mathbb{R}$ with values in $\mathbb{R}^{+}$, related to its autocorrelation function $R_{Z_{b}}(\eta)=E\left\{Z_{b}(x+\eta) Z_{b}(x)\right\}$ by the equation

$$
R_{Z_{b}}(\eta)=\int_{\mathbb{R}} e^{i k \eta} S_{Z_{b}}(k) d k
$$

Power spectral density function is defined by

$$
S_{Z_{b}}(k)=\frac{L}{\pi a} \frac{1}{\left(1+L^{2} k^{2}\right)} \mathbb{1}_{[-b, b]}(k)
$$

in which $0<b<+\infty$ is a finite positive real constant and where $a$ is such that $a=$ $\frac{2}{\pi} \arctan (b L)$. It can then verified that

$$
\sigma_{Y}^{2}=E\left\{Y(x)^{2}\right\}-1=\frac{2}{m_{Y}} \quad, \quad \sigma_{T}^{2}=E\left\{T(x)^{2}\right\}-1=\frac{2}{m_{T}} .
$$

\subsection{CONSTRUCTING THE RANDOM REDUCED MODEL}

The random reduced model of dimension $n$ is obtained by using the $n$ elastic modes $\underline{\varphi}_{1}, \ldots, \underline{\varphi}_{n}$ introduced in Section 2.4, associated with the $n$ lowest eigenfrequencies $\underline{\omega}_{1}, \ldots, \underline{\omega}_{n}$ defined by Eq. (47). From Eq. (57), it can be deduced that the approximation $U_{n}(x, \omega)$ of $U(x, \omega)$ is written as

$$
\begin{aligned}
& U_{n}(x, \omega)=\sum_{\alpha=1}^{n} Q_{\alpha}(\omega) \underline{\varphi}_{\alpha}(x) \quad, \quad x \in \underline{\Omega}, \\
& \left(-\omega^{2}\left[\underline{M}_{n}\right]+i \omega\left[\underline{D}_{n}\right]+\left[\mathbf{K}_{n}^{\mathrm{param}}\right]\right) \mathbf{Q}(\omega)=\mathbf{f}(\omega),
\end{aligned}
$$

in which $\left[\underline{M}_{n}\right]=\left[I_{n}\right]$ (see Eq. (46)), $\left[\underline{D}_{n}\right]=2 \underline{\xi} \omega_{\text {ref }}\left[I_{n}\right]$ (see Eq. (50)) and where $\mathbf{Q}(\omega)=$ $\left(Q_{1}(\omega), \ldots, Q_{n}(\omega)\right)$ is the random vector of the generalized coordinates and where $\mathbf{f}(\omega)=$ $\left(f_{1}(\omega), \ldots, f_{n}(\omega)\right)$ is the complex vector of the generalized external forces such that $f_{\alpha}(\omega)=$ $\int_{-1}^{+1} g(x, \omega) \underline{\varphi}_{\alpha}(x) d x$. Let $\left[\underline{K}_{n}\right]_{\alpha \beta}=\underline{\omega}_{\alpha}^{2} \delta_{\alpha \beta}$ be the matrix defined by Eq. (50) which can be written as

$$
\left[\underline{K}_{n}\right]=\left[\underline{L}_{K_{n}}\right]^{T}\left[\underline{L}_{K_{n}}\right] \quad, \quad\left[\underline{L}_{K_{n}}\right]_{\alpha \beta}=\underline{\omega}_{\alpha} \delta_{\alpha \beta}
$$

In Eq. (64), the random matrix $\left[\mathbf{K}_{n}^{\text {param }}\right]$ can be written as

$$
\left[\mathbf{K}_{n}^{\mathrm{param}}\right]=\left[\underline{L}_{K_{n}}\right]^{T}\left[\mathbf{G}_{K_{n}}^{\mathrm{param}}\right]\left[\underline{L}_{K_{n}}\right]
$$

where the random matrix $\left[\mathbf{G}_{K_{n}}^{\text {param }}\right]$ is such that

$$
\left[\mathbf{G}_{K_{n}}^{\mathrm{param}}\right]_{\alpha \beta}=\frac{\underline{k}_{1}}{\underline{\omega}_{\alpha} \underline{\omega}_{\beta}} \int_{-1}^{+1} Y(x) \underline{\varphi}_{\alpha}^{\prime \prime}(x) \underline{\varphi}_{\beta}^{\prime \prime}(x) d x+\frac{\underline{k}_{2}}{\underline{\omega}_{\alpha} \underline{\omega}_{\beta}} \int_{-1}^{+1} T(x) \underline{\varphi}_{\alpha}(x) \underline{\varphi}_{\beta}(x) d x .
$$


From Eqs. (58), (47) and (65), one deduces that

$$
\left[\underline{G}_{K_{n}}^{\text {param }}\right]=E\left\{\left[\mathbf{G}_{K_{n}}^{\text {param }}\right]\right\}=\left[I_{n}\right]
$$

In order to compare the non-parametric model with the parametric model, one introduces the global dispersion parameter $\delta_{K}^{\text {param }}>0$ of random matrix $\left[\mathbf{G}_{K_{n}}^{\text {param }}\right]$ defined (see Eqs. (3) to (6)) by

$$
\delta_{K}^{\mathrm{param}}=\left\{\frac{E\left\{\left\|\left[\mathbf{G}_{K_{n}}^{\mathrm{param}}\right]-\left[\underline{G}_{K_{n}}^{\mathrm{param}}\right]\right\|_{F}^{2}\right\}}{\left\|\left[\underline{G}_{K_{n}}^{\mathrm{param}}\right]\right\|_{F}^{2}}\right\}^{1 / 2} .
$$

The random generalized frequency response function associated with Eq. (64) is written as

$$
\left[\mathbf{H}_{n}(\omega)^{\text {param }}\right]=\left(-\omega^{2}\left[\underline{M}_{n}\right]+i \omega\left[\underline{D}_{n}\right]+\left[\mathbf{K}_{n}^{\text {param }}\right]\right)^{-1} .
$$

Finally, the random observation defined by Eq. (53) is written as

$$
\mathcal{E}_{n}^{\text {param }}(\omega)=\left\|\omega^{2}\left[\mathbf{H}_{n}^{\text {param }}(\omega)\right]\right\|_{F}
$$

\subsection{NUMERICAL ANALYSIS}

Statistics related to stochastic process $\left\{\mathcal{E}_{n}^{\text {param }}(\omega), \omega \in\left[0, \omega_{\max }\right]\right\}$ are estimated by using the Monte Carlo numerical simulation. Let $N$ be a fixed integer sufficiently high. It is assumed that $b=N \pi / 2$. Domain $[-1,+1]$ is discretized with the sampling space step $\Delta=2 / N$. In Eqs. (67), the integrals are discretized as follows,

$$
\left[\mathbf{G}_{K_{n}}^{\mathrm{param}}\right] \simeq \Delta \sum_{\nu=0}^{N-1}\left\{\frac{\underline{k}_{1}}{\underline{\omega}_{\alpha} \underline{\omega}_{\beta}} Y\left(x_{\nu}\right) \underline{\varphi}_{\alpha}^{\prime \prime}\left(x_{\nu}\right) \underline{\varphi}_{\beta}^{\prime \prime}\left(x_{\nu}\right)+\frac{\underline{k}_{2}}{\underline{\omega}_{\alpha} \underline{\omega}_{\beta}} T\left(x_{\nu}\right) \underline{\varphi}_{\alpha}\left(x_{\nu}\right) \underline{\varphi}_{\beta}\left(x_{\nu}\right)\right\},
$$

in which the sampling points in the space domain are

$$
x_{\nu}=-1+\nu \Delta \quad, \quad \nu=0,1, \ldots, N-1,
$$

and where $Y\left(x_{\nu}\right)$ and $T\left(x_{\nu}\right)$ are derived from Eq. (59). From Section 4.2, one then has to compute independent realizations of stochastic process $Z_{b}$. For $N$ fixed, one wishes stochastic process $Z_{b}$ to be Gaussian. Consequently, one uses the approximation $Z_{b}^{N}(x)$ of $Z_{b}(x)$ defined by

$$
Z_{b}^{N}(x)=\sqrt{2 \delta} \operatorname{Re}\left\{\sum_{\ell=0}^{N-1} \sqrt{S_{Z_{b}}\left(k_{\ell}\right)} R_{\ell} e^{-2 i \pi \cup_{\ell}-i k_{\ell} x}\right\} \quad, \quad R_{\ell}=\sqrt{-\ln \mathbb{V}_{\ell}},
$$


in which $\operatorname{Re}\{z\}$ denotes the real part of the complex number $z$ and where $\delta=2 b / N$ is the sampling wave number step such that $\delta \times \Delta=2 \pi / N$ (that is to say, $\delta=\pi$ ). The sampling points in the wave number domain are such that

$$
k_{\ell}=-b+\left(\ell+\frac{1}{2}\right) \delta \quad, \quad \ell=0,1, \ldots, N-1 .
$$

In Eq. (74), $\mathbb{U}_{0}, \mathbb{U}_{1}, \ldots, \mathbb{U}_{N-1}$ and $\mathbb{V}_{0}, \mathbb{V}_{1}, \ldots, \mathbb{V}_{N-1}$ are $2 N$ independent uniform realvalued random variables on $[0,1]$. With this choice of the parameter values, the FFT algorithm can be used to compute the independent realizations of the $\mathbb{R}^{N}$-valued random vector $\left(Z_{b}^{N}\left(x_{0}\right), \ldots, Z_{b}^{N}\left(x_{N-1}\right)\right)$.

\subsection{NUMERICAL PARAMETERS AND COMPUTATION}

The Monte Carlo numerical simulation method is carried out with $n_{s}=40000$ realizations, denoted $\theta_{1}, \ldots \theta_{n_{s}}$. The realizations $\omega \mapsto \mathcal{E}_{n}^{\text {param }}\left(\omega ; \theta_{1}\right), \ldots, \omega \mapsto \mathcal{E}_{n}^{\text {param }}\left(\omega ; \theta_{n_{s}}\right)$ are numerically calculated on the frequency band $\left[0, \omega_{\max }\right]$ with $\omega_{\max }=2 \pi \times 0.22 \mathrm{rad} / \mathrm{s}$ and with a sampling frequency step $\Delta \omega=\omega_{\max } / 300$. The values of the numerical parameters are $n=30$, $m_{Y}=m_{T}=4, N=512, L=0.076 m, \delta=\pi=3.1415 m^{-1}, \Delta=2 / N=0.0039 m$, $b=N \pi / 2=804.25 \mathrm{~m}^{-1}$ and $a=0.9896$. Figure 11 on the left displays the graph of power spectral density function $k \mapsto S_{Z_{b}}(k)$ and Figure 11 on the right displays the graph of autocorrelation function $\eta \mapsto R_{Z_{b}}(k)$. The computation of parameter $\delta_{K}^{\text {param }}$ defined by Eq. (69) yields $\delta_{K}^{\text {param }}=0.4942 \simeq 0.50$.

Figures 12 and 13 are relative to the frequency band $[0,0.22] \mathrm{Hz}$. Figure 12 on the left displays the response $\nu \mapsto \log _{10} \underline{e}_{n}(2 \pi \nu)$ of the mean dynamic system (dashed line) calculated in Section 3.3 and the graph of function $\nu \mapsto \mathrm{dB}^{\text {param }}(\nu)$ (thick solid line) such that

$$
\mathrm{dB}^{\text {param }}(\nu)=\log _{10}\left(E\left\{\mathcal{E}_{n}^{\text {param }}(2 \pi \nu)\right\}\right)
$$

Figure 12 on the right displays the graph of the function $\nu \mapsto \sigma_{\mathcal{E}_{n}^{\text {param }}}(2 \pi \nu)$ (solid line) in which $\sigma_{\mathcal{E}_{n}^{\text {param }}}(\omega)$ is the standard deviation of random variable $\mathcal{E}_{n}^{\text {param }}(\omega)$. Figure 13 displays the graphs of functions $\nu \mapsto \mathrm{dB}^{\text {param }}(\nu)$ (dashed line), $\nu \mapsto \mathrm{dB}_{\text {max }}^{\text {param }}(\nu)$ (upper solid line) and $\nu \mapsto \mathrm{dB}_{\min }^{\text {param }}(\nu)$ (lower solid line) in which

$$
\begin{aligned}
& \mathrm{dB}_{\text {max }}^{\text {param }}(\nu)=\log _{10}\left\{\max _{k} \mathcal{E}_{n}^{\text {param }}\left(2 \pi \nu ; \theta_{k}\right)\right\}, \\
& \mathrm{dB}_{\text {min }}^{\text {param }}(\nu)=\log _{10}\left\{\min _{k} \mathcal{E}_{n}^{\text {param }}\left(2 \pi \nu ; \theta_{k}\right)\right\} .
\end{aligned}
$$


Due to the fact that $\delta_{K}=0.50 \simeq \delta_{K}^{\text {param }}=0.4942$, one can compare the results given by the non-parametric approach (Figures 9 and 10) with the results given by the parametric approach (Figures 12 and 13). These figures cleary prove that the non-parametric results look like the parametric results when the "positive-definite" ensemble is used and is very different in the lowfrequency domain when the GOE is used. Consequently, the present results give an additional validation point of the non-parametric model of random uncertainties for which the theory is recalled in Sections 1.1 and 1.2 and which is based on the "positive-definite" ensemble.

\section{CONCLUSIONS}

This paper gives a new validation point of the non-parametric theory of random uncertainties in vibration analysis, recently introduced by the author. It is proved that the "positive-definite" ensemble of random matrices, which has been introduced in the context of the development of this non-parametric approach, is well adapted to the low-frequency vibration analysis, while the use of the Gaussian orthogonal ensemble (GOE) is not, particularly for strong values of the dispersion parameter. In addition, as it is explained in previous papers devoted to the construction of this non-parametric approach, the "positive-definite" ensemble allows random uncertainties to be modelled for the damping operator while the GOE does not, in the present state of the art.

\section{REFERENCES}

1. E. P. Wigner 1951 Proc. Cambridge Philos. Soc. 47, 790-798. On the statistical distribution of the widths and spacings of nuclear resonance levels.

2. F. J. Dyson 1962 J. Math. Phys. 3, 140-175. Statistical theory of the energy levels of complex systems. Parts I,II,III.

3. E. P. Wigner 1962 Distribution laws for the roots of a random Hermitian matrix, in Statistical Theories of Spectra: Fluctuations, edited by C. E. Poter, 1965, New York: Academic Press.

4. F. J. Dyson and M. L. Menta 1963 J. Math. Phys. 4, 701-719. Statistical theory of the energy levels of complex systems. Parts IV,V.

5. C. E. Poter 1965 Statistical Theories of Spectra: Fluctuations. New York: Academic Press. 
6. M. L. Menta 1967 Random Matrices and the Statisticals Theory of Energy Levels. New York: Academic Press.

7. M. L. Menta 1991 Random Matrices. Revised and Enlarged Second Edition. New York: Academic Press.

8. O. Bohigas, M.-J. Giannoni and C. Schmit 1984 Physical Review Letters 52(1), 1-4. Characterization of Chaotic Quantum Spectra and Universality of Level Fluctuation Laws.

9. O. Bohigas, M.-J. Giannoni And C. Schmit 1986 in Quantum Chaos and Statistical Nuclear Physics, edited by T. H. Seligman and H. Nishioka, New York: Springer, pp. 18-40. Spectral fluctuations of classically chaoctic quantum systems.

10. R. L. Weaver 1989 J. Acoust. Soc. Am. 85(3), 1005-1013. Spectral statistics in elastodynamics.

11. O. Legrand And D. Sornette 1990 Physica D 44, 229-235. Coarse-grained properties of the chaotic trajectories in the stadium.

12. O. Bohigas, O. Legrand, C. Schmit and D. Sornette 1991 J. Acoust. Soc. Am. 89(3), 1456-1458. Comment on spectral statistics in elastodynamics.

13. C. Schmit 1991 in Chaos and Quantum Physics, edited by M.-J. Giannoni, A. Voros and J. Zinn-Justin, Amsterdam: North-Holland, pp. 333-369. Quantum and classical properties of some billiards on the hyperbolic plane.

14. O. Legrand, C. Schmit And D. Sornette 1992 Europhysics Letters 18(2), 101-106. Quantum chaos methods applied to high-frequency plate vibrations.

15. R. S. Langley 1999 Proc. Roy. Soc. Series A 455, 3325-3349. A non-Poisson model for the vibration analysis of uncertain dynamic systems.

16. C. SoIzE 1999 Publication du LMA-CNRS ISBN 2-909669-16-5, 152, 109-138. A nonparametric model of random uncertainties in linear structural dynamics.

17. C. Solze 2000 Probabilistic Engineering Mechanics 15(3), 277-294. A nonparametric model of random uncertainties for reduced matrix models in structural dynamics.

18. C. Soize 2001 J. Acoust. Soc. Am. 109(5), 1979-1996. Maximum entropy approach for modeling random uncertainties in transient elastodynamics.

19. C. Solze 2001 Probabilistic Engineering Mechanics 6(4), 363-372. Transient responses of dynamical systems with random uncertainties.

20. C. Solze 2001 Uncertainties in Engineering Mechanics, 1(1), 1-38, e-journal from Resonance Publication (http://www.resonance-pub.com). Nonlinear dynamical systems with nonparametric model of random uncertainties.

21. E. J. Haug, K. K. Choi And V. Komkov 1986 Design Sensitivity Analysis of Structural 
Systems. San diego/London: Academic Press.

22. R. A. Ibrahim 1987 Applied Mechanics Reviews 40(3), 309-328. Structural dynamics with parameter uncertainties.

23. D. Iwan And H. Jensen 1993 Transactions of ASME 60, 484-490. On the dynamical response of continuous systems including model uncertainty.

24. C. LeE And R. Singh 1994 Journal of Sound and Vibration 174(3), 395-412. Analysis of discrete vibratory systems with parameter uncertainties. Part II: Impulse response.

25. E. Vanmarcke And M. Grigoriu 1983 Journal of Engineering Mechanics 109(5), 12031214. Stochastic finite element analysis of simple beams",

26. W. K. Liu, T. Belytschko And A. Mani 1986 International Journal of Numerical Methods in Engineering 23, 1832-1845. Random field finite elements.

27. M. Shinozuka And G. Deodatis 1988 Journal of Engineering Mechanics 114(3), 499519. Response variability of stochastic finite element systems.

28. P. D. Spanos And R. G. Ghanem 1989 ASCE Journal of Engineering Mechanics 115(5), 1035-1053. Stochastic finite element expansion for random media.

29. R. G. Ghanem And P. D. Spanos 1991 Stochastic Finite Elements: A Spectral Approach. New York: Springer-Verlag.

30. M. Kleiber And T. D. Hien 1992 The Stochastic Finite Element Method. Chichester: John Wiley and Sons.

31. C. E. Shannon 1948 Bell System Tech. J. 27, 379-423 and 623-659. A mathematical theory of communication.

32. E. T. Jaynes 1957 Physical Review 106(4), 620-630 and 108(2), 171-190. Information theory and statistical mechanics.

33. J. N. Kapur And H. K. Kesavan 1992 Entropy Optimization Principles with Applications. San Diego: Academic Press.

34. G. JUMARIE 2000 Maximum Entropy, Information Without Probability and Complex Fractals. Dordrecht/Boston/London: Kluwer Academic Publishers.

35. T.A. Brody 1973 Nuovo Cimento Lett. 7, 482-489. A statistical measure for the repulsion of energy levels.

36. R. Y. Rubinstein 1981 Simulation and the Monte Carlo Method. New York: John Wiley and Sons.

37. P. K. MacKeOwn 1997 Stochastic Simulation in Physics. Singapore: Springer-Verlag 


\section{CAPTIONS ACCOMPANYING EACH FIGURE}

Figure 1. Dispersion parameter $\delta_{K}=0.25$. Figure on the left: graphs of functions $j \mapsto m_{\Lambda_{j}}$ (thick solid line) and $j \mapsto m_{\Lambda_{j}^{\mathrm{GOE}}}$ (thin solid line). Figure on the right: graphs of functions $j \mapsto \sigma_{\Lambda_{j}}$ (thick solid line) and $j \mapsto \sigma_{\Lambda_{j}^{\mathrm{GOE}}}($ thin solid line).

Figure 2. Dispersion parameter $\delta_{K}=0.25$. Figure on the left: for $j=1, \ldots, 30$, graph of function $\lambda \mapsto p_{\Lambda_{j}^{\mathrm{GOE}}}(\lambda)$. Figure on the right: for $j=1, \ldots, 30$, graph of function $\lambda \mapsto p_{\Lambda_{j}}(\lambda)$.

Figure 3. Dispersion parameter $\delta_{K}=0.25$. Figure on the left: graphs of functions $j \mapsto m_{\Delta_{j}}$ (thick solid line) and $j \mapsto m_{\Delta_{j}^{\mathrm{GOE}}}$ (thin solid line). Figure on the right: graphs of functions $j \mapsto \sigma_{S_{j}}$ (thick solid line) and $j \mapsto \sigma_{S_{j}^{\mathrm{GOE}}}$ (thin solid line).

Figure 4-a. "Positive-definite" ensemble; dispersion parameter $\delta_{K}=0.25$. Figure on the left: for $j=1, \ldots, 30$, graph of function $s \mapsto \log _{10}\left(p_{S_{j}}(s)\right)$ (irregular solid lines) and graph of the Wigner pdf $s \mapsto \log _{10}\left(p_{W}(s)\right)$ (regular solid line). Figure on the right: graph of function $s \mapsto \log _{10}\left(p_{S}(s)\right)$ (irregular thick solid line), graph of the Wigner pdf $s \mapsto \log _{10}\left(p_{W}(s)\right)$ (dashed line) and graph of the Brody pdf $s \mapsto \log _{10}\left(p_{B}(s)\right)$ with $\alpha=1.93$ (thin solid line).

Figure 4-b. Gaussian orthogonal ensemble; dispersion parameter $\delta_{K}=0.25$. Figure on the left: for $j=1, \ldots, 30$, graph of function $s \mapsto \log _{10}\left(p_{S_{j}}^{\mathrm{GOE}}(s)\right)$ (irregular solid lines) and graph of the Wigner pdf $s \mapsto \log _{10}\left(p_{W}(s)\right)$ (regular solid line). Figure on the right: graph of function $s \mapsto \log _{10}\left(p_{S}^{\mathrm{GOE}}(s)\right)$ (irregular thick solid line), graph of the Wigner pdf $s \mapsto \log _{10}\left(p_{W}(s)\right)$ (dashed line) and graph of the Brody pdf $s \mapsto \log _{10}\left(p_{B}(s)\right)$ with $\alpha=1.93$ (thin solid line).

Figure 5. Dispersion parameter $\delta_{K}=0.50$. Figure on the left: graphs of functions $j \mapsto m_{\Lambda_{j}}$ (thick solid line) and $j \mapsto m_{\Lambda_{j}^{\mathrm{GOE}}}$ (thin solid line). Figure on the right: graphs of functions $j \mapsto \sigma_{\Lambda_{j}}$ (thick solid line) and $j \mapsto \sigma_{\Lambda_{j}^{\mathrm{GOE}}}$ (thin solid line).

Figure 6. Dispersion parameter $\delta_{K}=0.50$. Figure on the left: for $j=1, \ldots, 30$, graph of function $\lambda \mapsto p_{\Lambda_{j}^{\mathrm{GOE}}}(\lambda)$. Figure on the right: for $j=1, \ldots, 30$, graph of function $\lambda \mapsto p_{\Lambda_{j}}(\lambda)$.

Figure 7. Dispersion parameter $\delta_{K}=0.50$. Figure on the left: graphs of functions $j \mapsto m_{\Delta_{j}}$ (thick solid line) and $j \mapsto m_{\Delta_{j}^{\mathrm{GOE}}}$ (thin solid line). Figure on the right: graphs of functions $j \mapsto \sigma_{S_{j}}$ (thick solid line) and $j \mapsto \sigma_{S_{j}^{\mathrm{GOE}}}$ (thin solid line).

Figure 8-a. "Positive-definite" ensemble; dispersion parameter $\delta_{K}=0.50$. Figure on the left: for $j=1, \ldots, 30$, graph of function $s \mapsto \log _{10}\left(p_{S_{j}}(s)\right)$ (irregular solid lines) and graph of 
the Wigner pdf $s \mapsto \log _{10}\left(p_{W}(s)\right)$ (regular solid line). Figure on the right: graph of function $s \mapsto \log _{10}\left(p_{S}(s)\right)$ (irregular thick solid line), graph of the Wigner pdf $s \mapsto \log _{10}\left(p_{W}(s)\right)$ (dashed line) and graph of the Brody pdf $s \mapsto \log _{10}\left(p_{B}(s)\right)$ with $\alpha=1.91$ (thin solid line).

Figure 8-b. Gaussian orthogonal ensemble; dispersion parameter $\delta_{K}=0.50$. Figure on the left: for $j=1, \ldots, 30$, graph of function $s \mapsto \log _{10}\left(p_{S_{j}}^{\mathrm{GoE}}(s)\right)$ (irregular solid lines) and graph of the Wigner pdf $s \mapsto \log _{10}\left(p_{W}(s)\right)$ (regular solid line). Figure on the right: graph of function $s \mapsto \log _{10}\left(p_{S}^{\mathrm{GOE}}(s)\right)$ (irregular thick solid line), graph of the Wigner pdf $s \mapsto \log _{10}\left(p_{W}(s)\right.$ ) (dashed line) and graph of the Brody pdf $s \mapsto \log _{10}\left(p_{B}(s)\right)$ with $\alpha=1.91$ (thin solid line).

Figure 9. Non-parametric approach. Dispersion parameter $\delta_{K}=0.50$. Frequency band $[0,0.22] H z$ (horizontal axis). Figure on the left: graphs of functions $\nu \mapsto \log _{10} \underline{e}_{n}(2 \pi \nu)$ (dashed line), $\nu \mapsto \mathrm{dB}(\nu)$ (thick solid line) and $\nu \mapsto \mathrm{dB}^{\mathrm{GOE}}(\nu)$ (thin solid line). Figure on the right: graphs of functions $\nu \mapsto \sigma_{\mathcal{E}_{n}}(2 \pi \nu)$ (thick solid line) and $\nu \mapsto \sigma_{\mathcal{E}_{n}^{\mathrm{GOE}}}(2 \pi \nu)$ (thin solid line).

Figure 10. Non-parametric approach. Dispersion parameter $\delta_{K}=0.50$. For the "positivedefinite" ensemble: graphs of functions $\nu \mapsto \mathrm{dB}(\nu)$ (thick dashed line), $\nu \mapsto \mathrm{dB}_{\max }(\nu)$ (upper thick solid line), $\nu \mapsto \mathrm{dB}_{\min }(\nu)$ (lower thick solid line). For the Gaussian orthogonal ensemble, graphs of functions $\nu \mapsto \mathrm{dB}^{\mathrm{GOE}}(\nu)$ (thin dashed line), $\nu \mapsto \mathrm{dB}_{\max }^{\mathrm{GOE}}(\nu)$ (upper thin solid line), $\nu \mapsto \mathrm{dB}_{\min }^{\mathrm{GOE}}(\nu)$ (lower thin solid line).

Figure 11. Figure on the left: graph of power spectral density function $k \mapsto S_{Z_{b}}(k)$. Figure on the right: graph of autocorrelation function $\eta \mapsto R_{Z_{b}}(k)$.

Figure 12. Parametric approach. Dispersion parameter $\delta_{K}^{\text {param }}=0.4942$. Frequency band $[0,0.22] H z$ (horizontal axis). Figure on the left: graphs of functions $\nu \mapsto \log _{10} \underline{e}_{n}(2 \pi \nu)$ (dashed line) and $\nu \mapsto \mathrm{dB}^{\text {param }}(\nu)$ (thick solid line). Figure on the right: graph of function $\nu \mapsto \sigma_{\mathcal{E}_{n}^{\text {param }}}(2 \pi \nu)$ (solid line).

Figure 13. Parametric approach. Dispersion parameter $\delta_{K}^{\text {param }}=0.4942$. Frequency band $[0,0.22] \mathrm{Hz}$ (horizontal axis). Graphs of functions $\nu \mapsto \mathrm{dB}^{\text {param }}(\nu)$ (dashed line), $\nu \mapsto$ $\mathrm{dB}_{\max }^{\text {param }}(\nu)$ (upper solid line) and $\nu \mapsto \mathrm{dB}_{\min }^{\text {param }}(\nu)$ (lower solid line). 

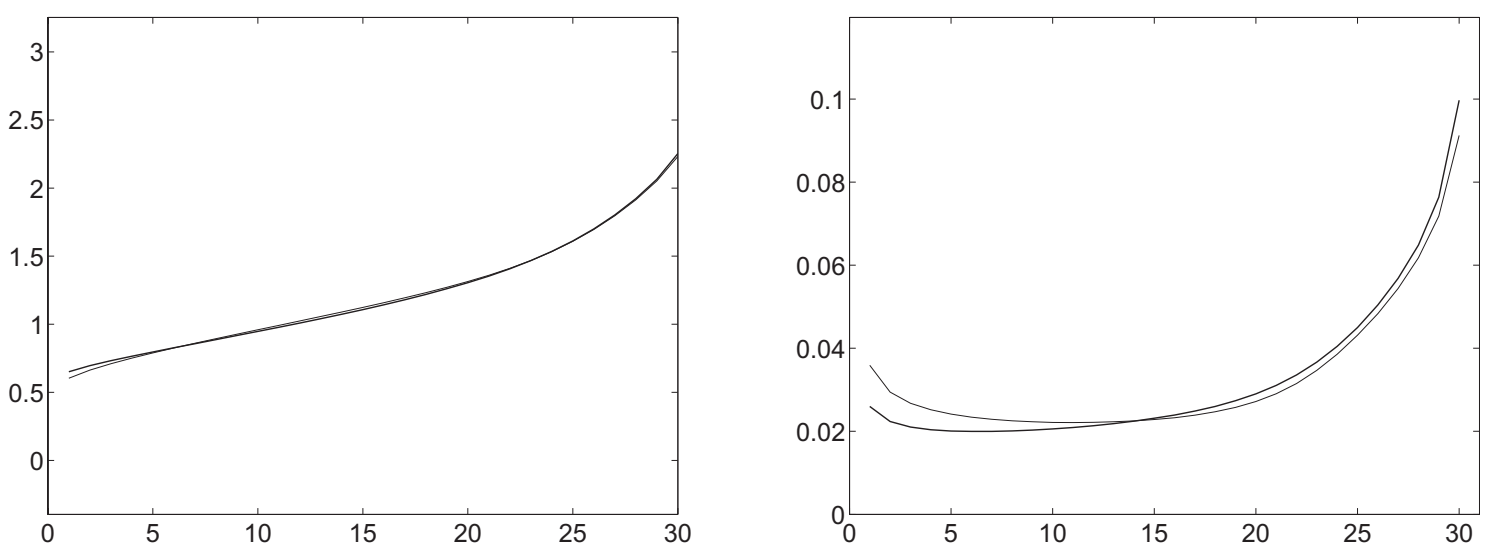

Figure 1 (left and right)
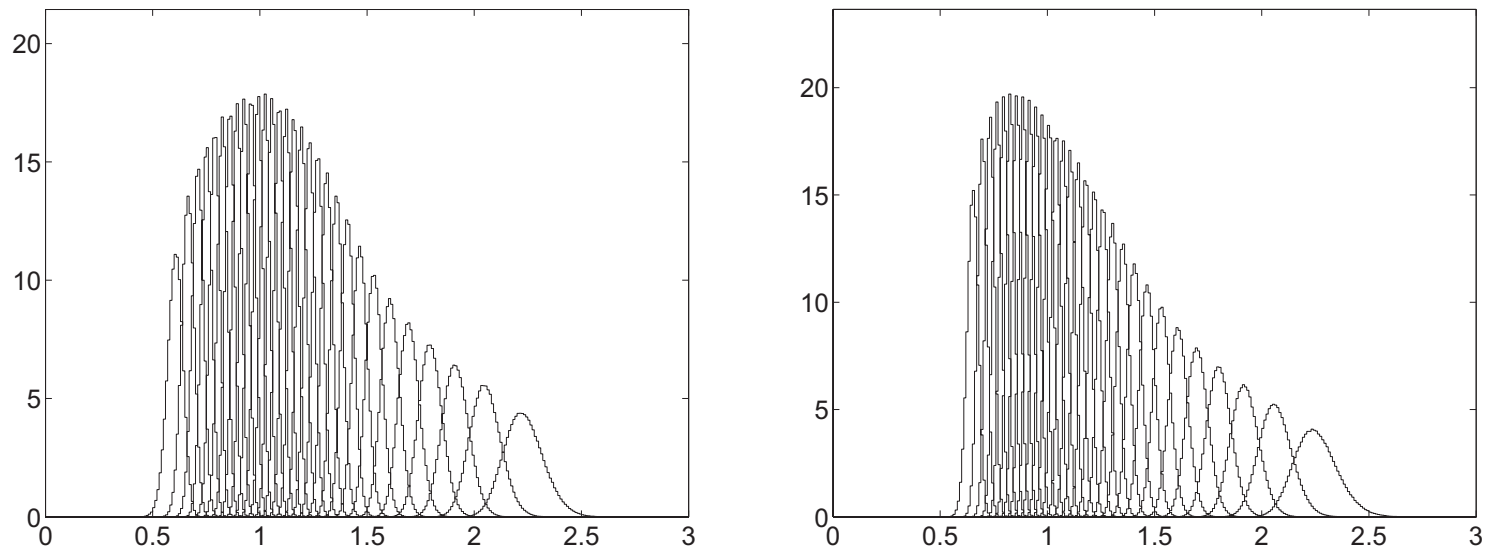

Figure 2 (left and right)
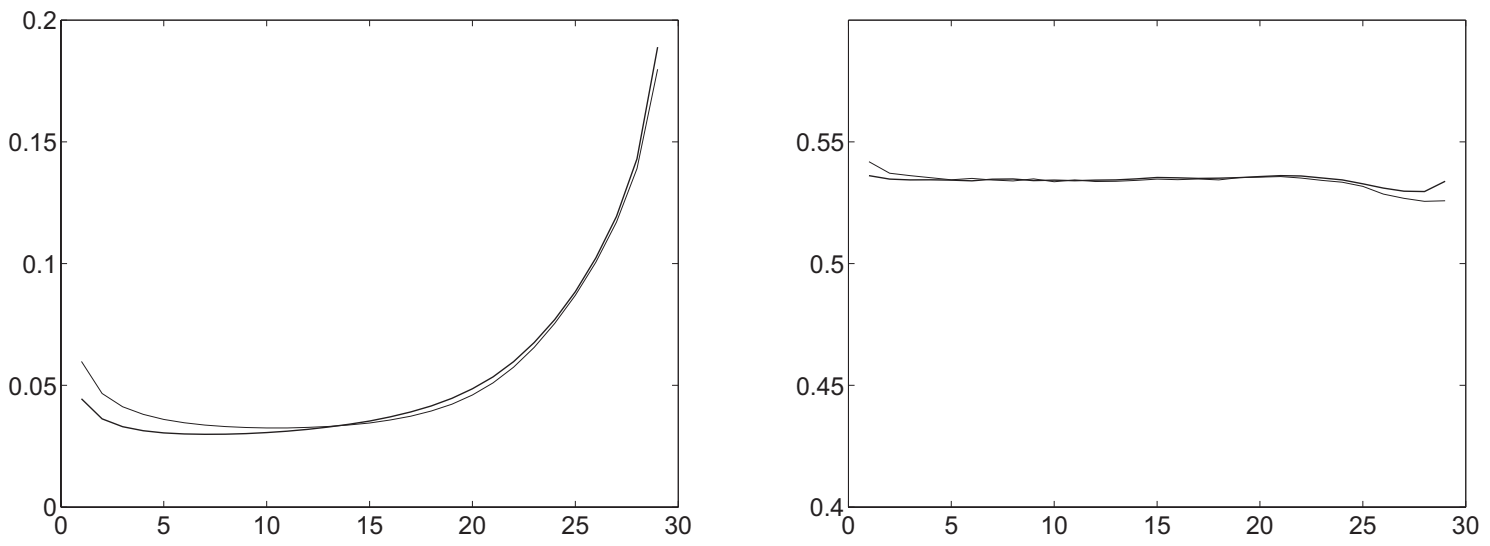

Figure 3 (left and right) 

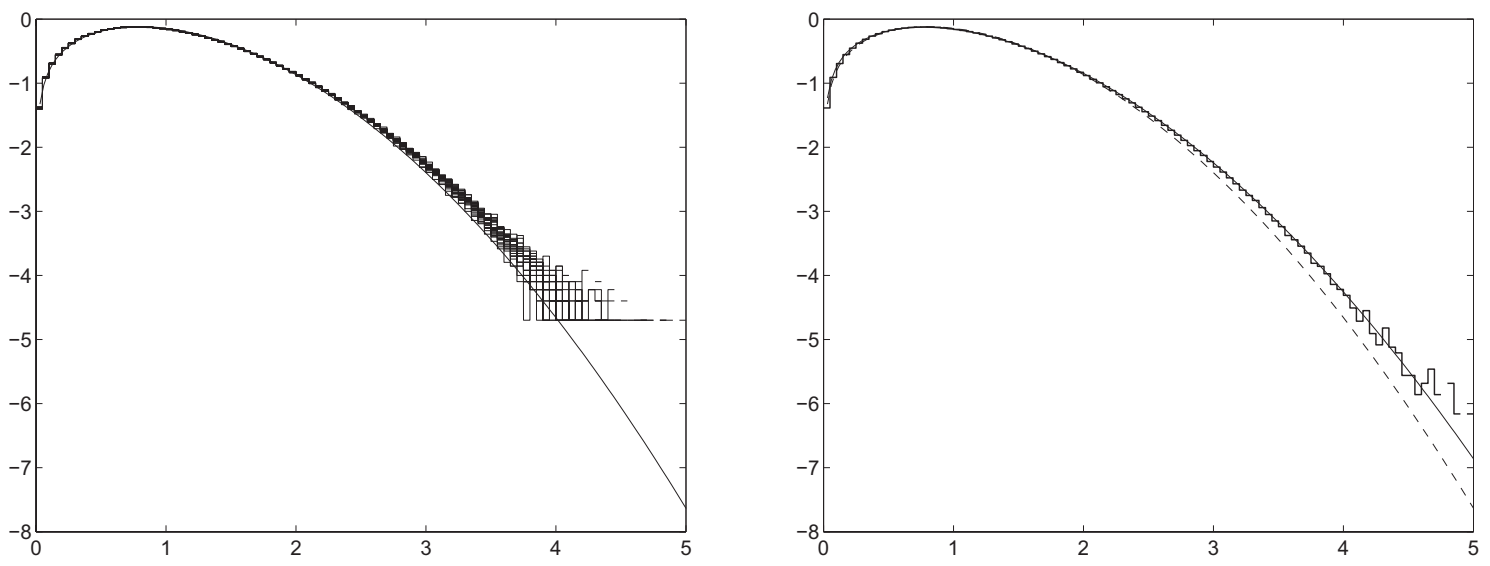

Figure 4-a (left and right)
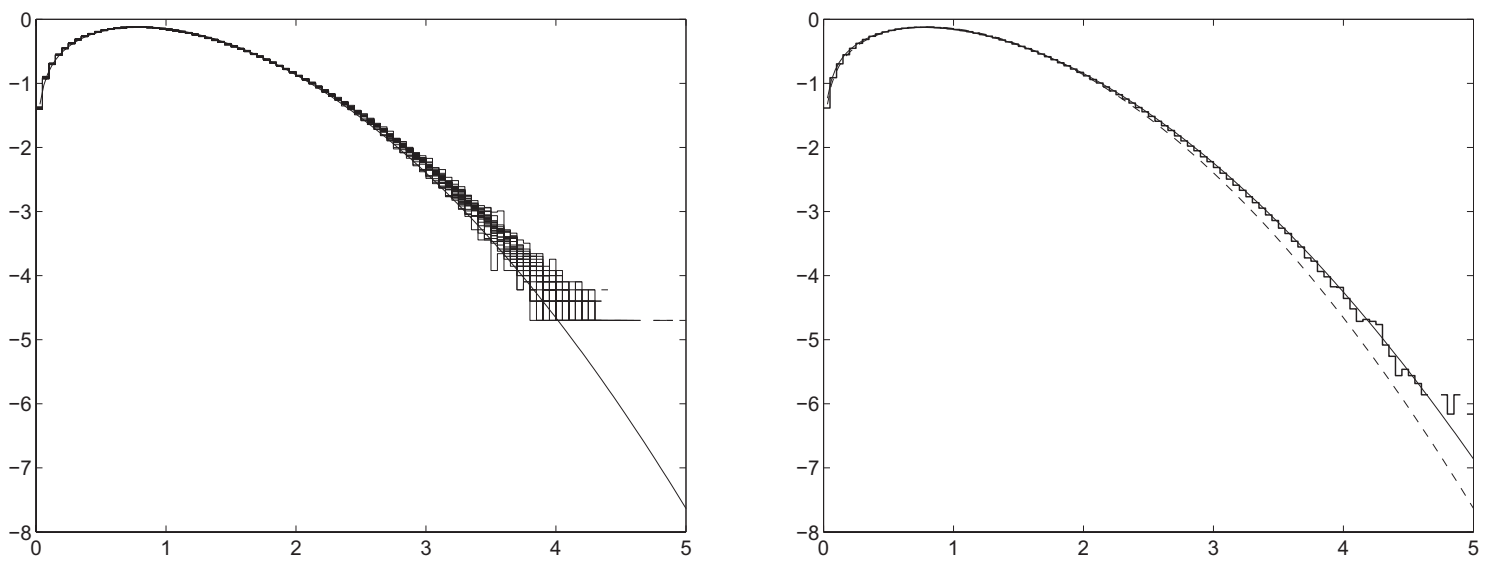

Figure 4-b (left and right)
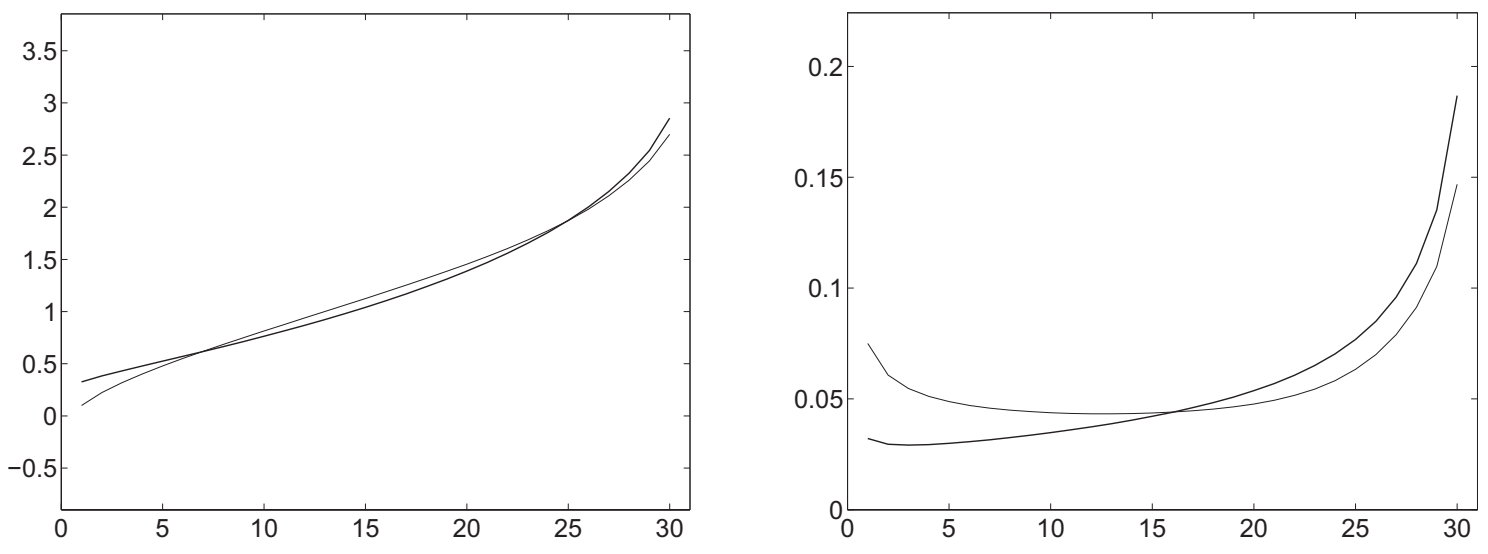

Figure 5 (left and right) 

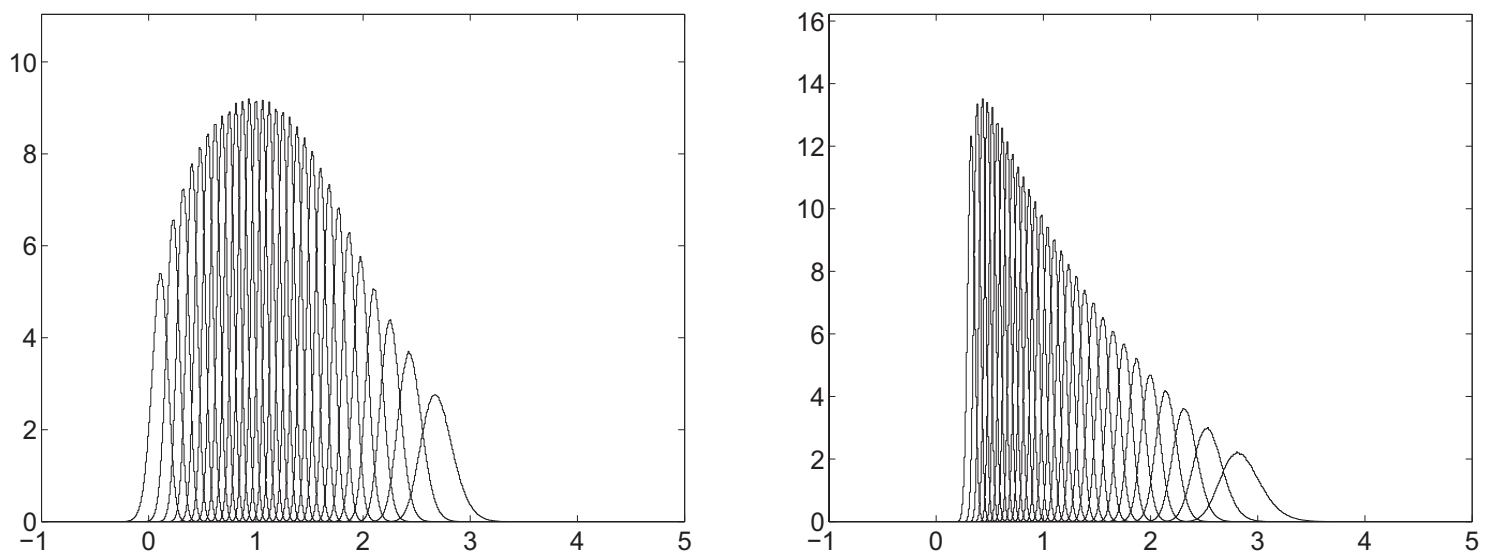

Figure 6 (left and right)
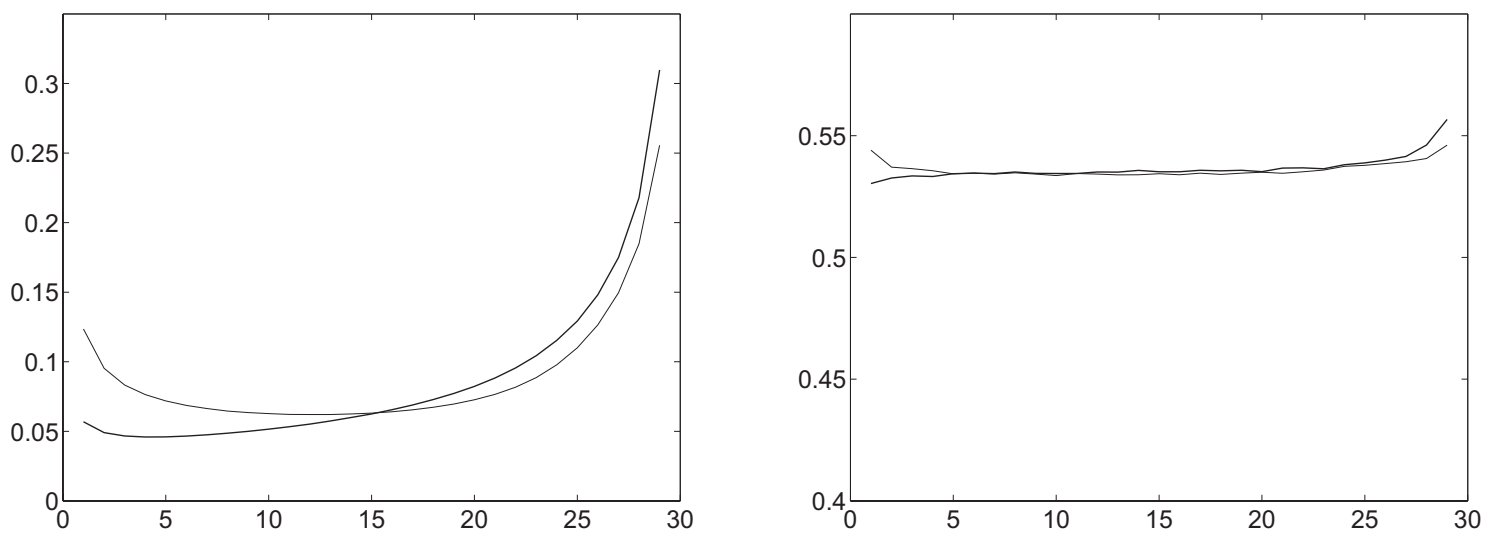

Figure 7 (left and right)
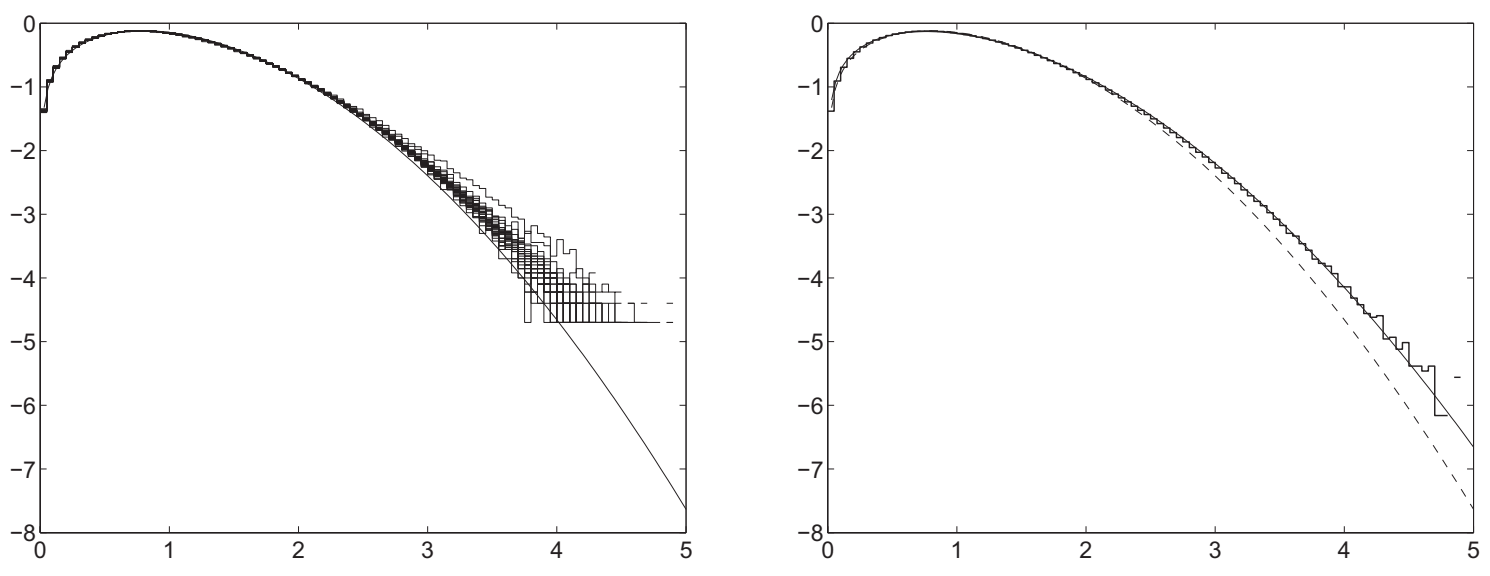

Figure 8-a (left and right) 

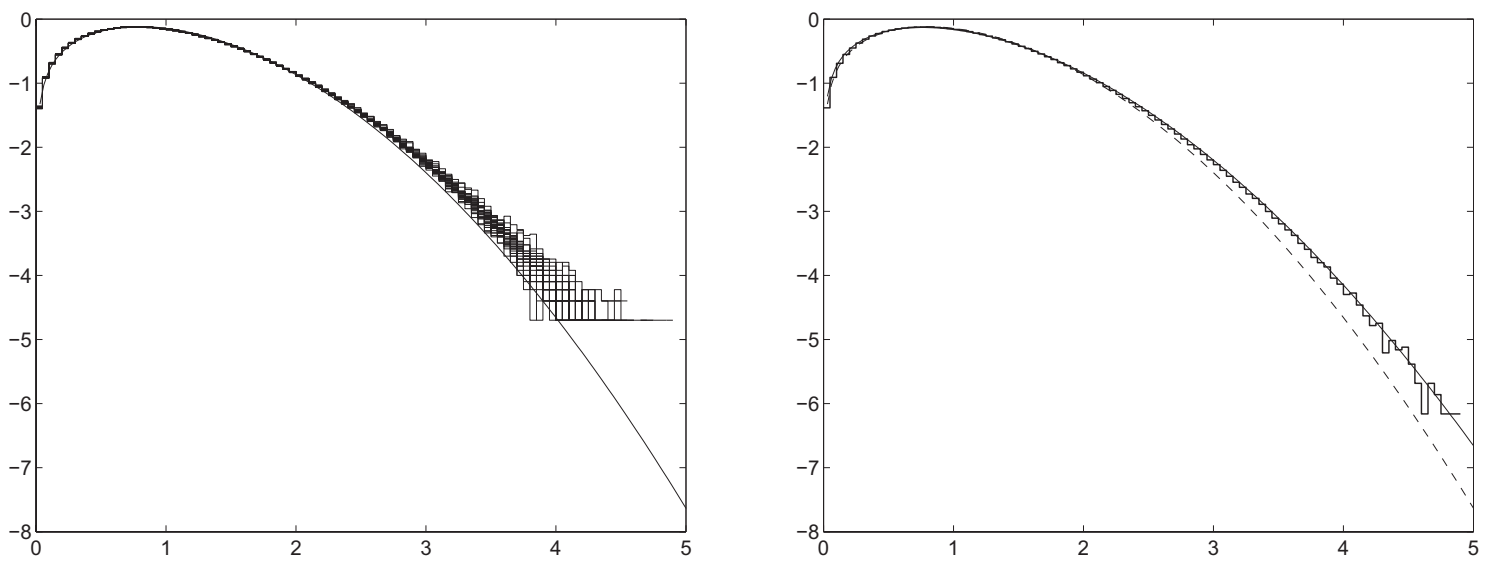

Figure 8-b (left and right)
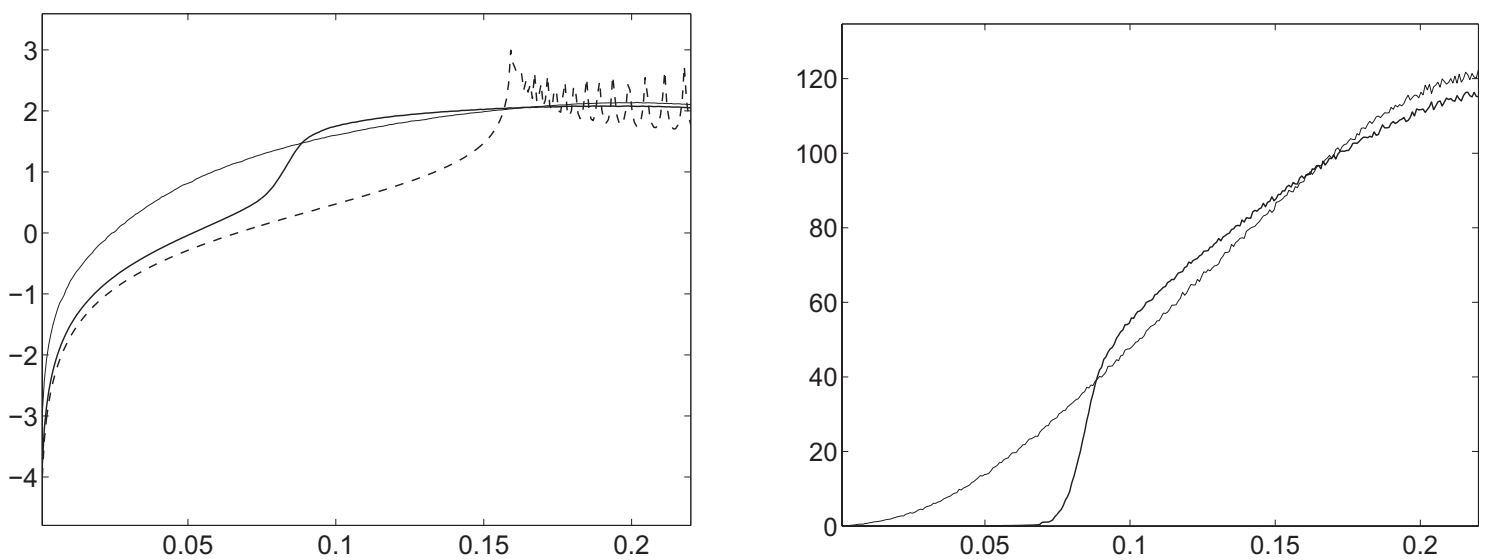

Figure 9 (left and right)

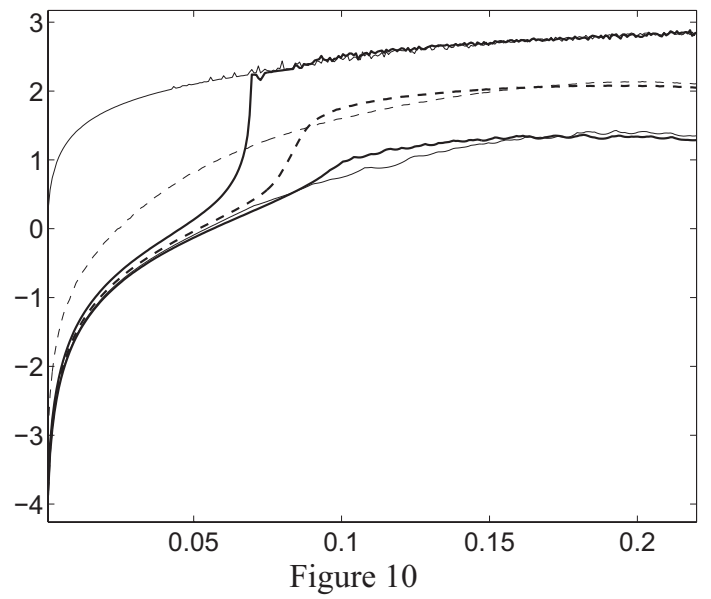



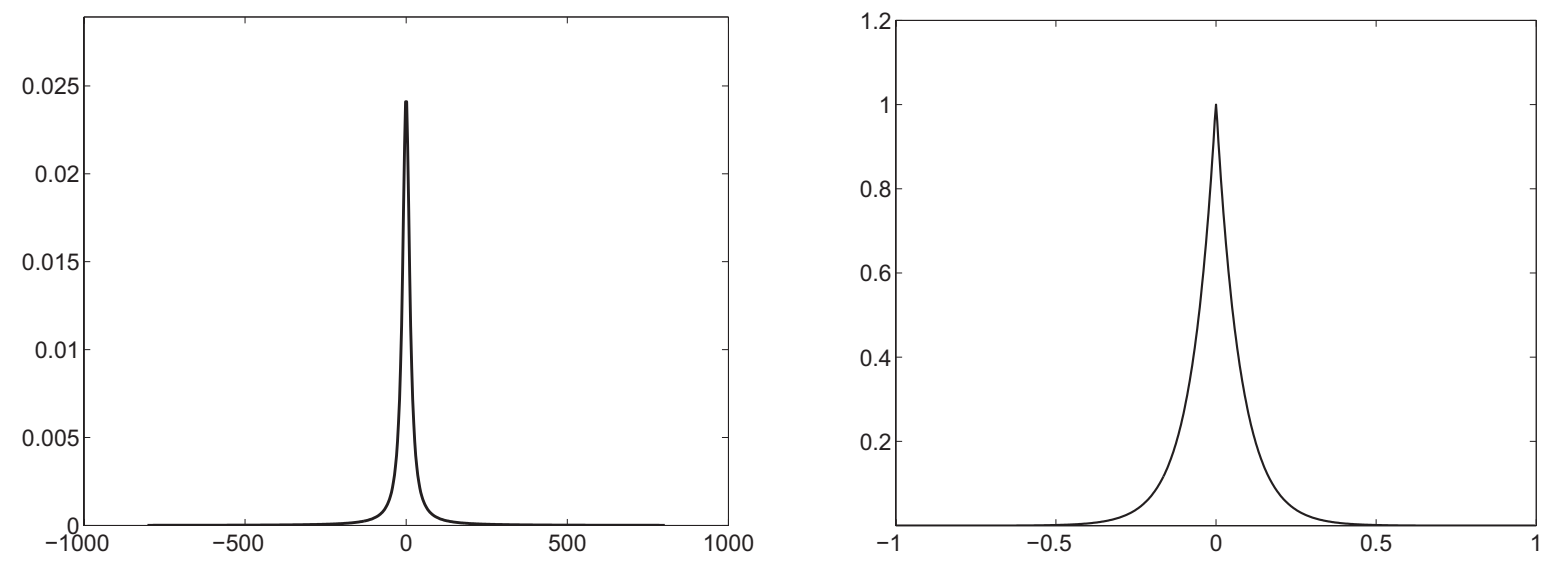

Figure 11 (left and right)
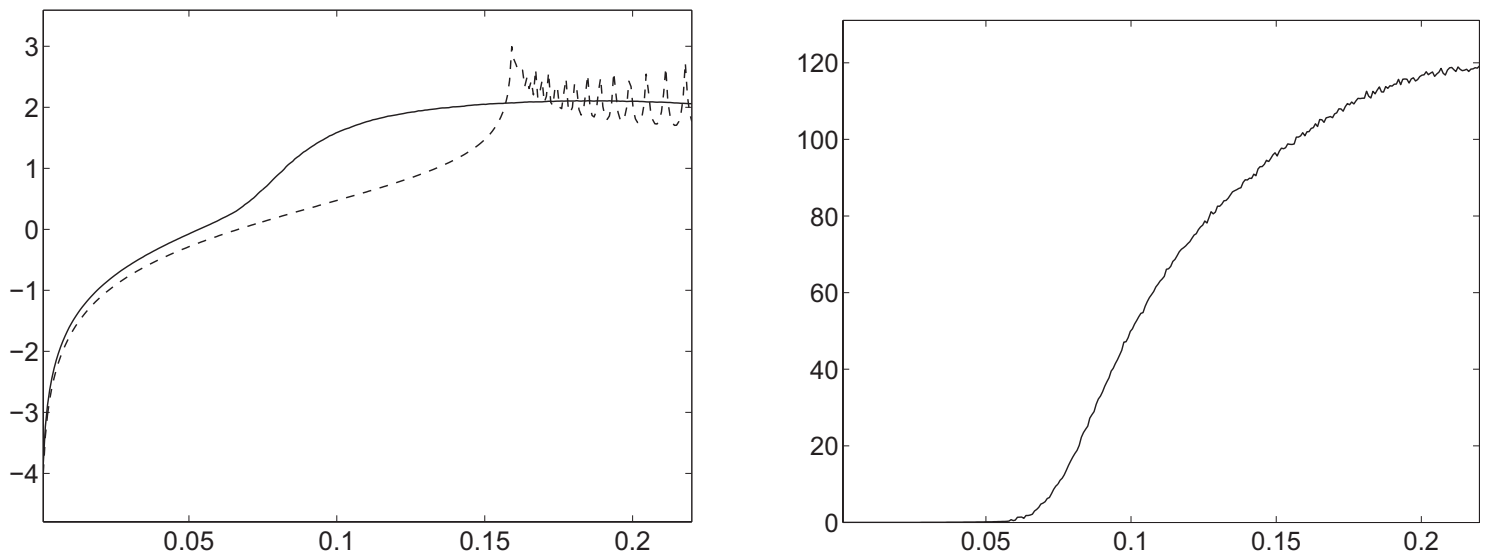

Figure 12 (left and right)

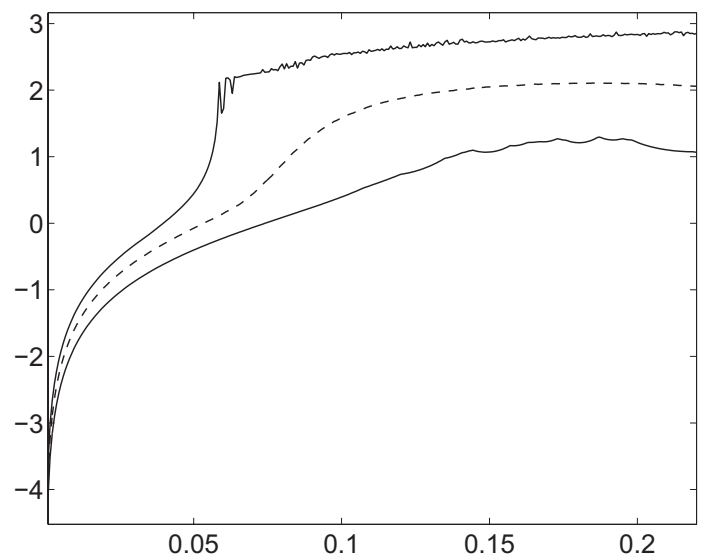

Figure 13 